\title{
Front propagation into unstable states: Marginal stability as a dynamical mechanism for velocity selection
}

\author{
Wim van Saarloos \\ AT\&T Bell Laboratories, Murray Hill, New Jersey 07974-2070
}

(Received 27 May 1987)

\begin{abstract}
In this paper the propagation of fronts into an unstable state are studied. Such fronts can occur e.g., in the form of domain walls in liquid crystals, or when the dynamics of a system which is suddenly quenched into an unstable state is dominated by domain walls moving in from the boundary. It was emphasized recently by Dee et al. that for sufficiently localized initial conditions the velocity of such fronts often approaches the velocity corresponding to the marginal stability point, the point at which the stability of a front profile moving with a constant speed changes. I show here when and why this happens, and advocate the marginal stability approach as a simple way to calculate the front velocity explicitly in the relevant cases. I sketch the physics underlying this dynamical mechanism with analogies and, building on recent work by Shraiman and Bensimon, show how an equation for the local "wave number" that may be viewed as a generalization of the Burgers equation, drives the front velocity to the marginal stability value. This happens provided the steady-state solutions lose stability because the group velocity for perturbations becomes larger than the envelope velocity of the front. For a given equation, our approach allows one to check explicitly that the marginal stability fixed point is attractive, and this is done for the amplitude equation and the Swift-Hohenberg equation. I also analyze an extension of the FisherKolmogorov equation, obtained by adding a stabilizing fourth-order derivative $-\gamma \partial^{4} \phi / \partial x^{4}$ to it. I predict that for $\gamma<\frac{1}{12}$ the fronts in this equation are of the same type as those occurring in the Fisher-Kolmogorov equation, i.e., localized initial conditions develop into a uniformly translating front solution of the form $\phi(x-v t)$ that propagates with the marginal stability velocity. For $\gamma>\frac{1}{12}$, localized initial conditions may develop into fronts propagating at the marginal stability velocity, but such front solutions cannot be uniformly translating. Differences between the propagation of uniformly translating fronts $\phi(x-v t)$ and envelope fronts are pointed out, and a number of open problems, some of which could be studied numerically, are also discussed.
\end{abstract}

\section{INTRODUCTION}

In this paper ${ }^{1(a)}$ we will discuss the dynamics of fronts or domain walls which are propagating into an unstable state. The approach we will develop applies to the type of equation that often arises in the analysis of systems that are suddenly quenched into an unstable state, and whose subsequent time evolution is dominated by the propagation of well-developed fronts or domain walls (an illustrative example of such dynamics will be discussed in Sec. II). Problems of this type have been studied since the $1930 \mathrm{~s}^{1(\mathrm{~b}), 2}$ in biology; they arise, e.g., in discussions of population dynamics ${ }^{3}$ and pulse propagation in nerves. ${ }^{4}$ The interest in physics in this type of front propagation has been growing only recently, mainly as a result of two stimulating papers by Dee et al. ${ }^{5,6}$ Physical examples of fronts propagating into an unstable state occur, e.g., in fluid flow instabilities ${ }^{7}$ when the system is suddenly brought above the instability threshold. ${ }^{8}$ For instance, if in the experiments by Ahlers and Cannell ${ }^{9}$ on the Taylor-Couette system, the Couette flow is suddenly made unstable, Taylor vortices first grow near the top and bottom edges of the system. This is followed by the propagation of a "vortex front" into the bulk. In such experiments, the propagation of the front determines the wavelength of the state emerging behind it, and thus it leads to a form of pattern selection. ${ }^{7}$ Other examples in physics of the type of problems we discuss arise in studies of the propagation of domain walls in liquid crystals ${ }^{10-12}$ (see also Sec. II), in traveling waves in reaction-diffusion systems, ${ }^{7,13}$ excitable media ${ }^{14}$ such as chemical reactions, ${ }^{13-16}$ and in combustion, ${ }^{17}$ as well as in mean-field models of aggregation ${ }^{18-20}$ and deposition. $^{21}$ In some of these examples, the state emerging behind the front is relatively featureless, so that the main quantity of interest is the propagation velocity.

The prototype equation to illustrate some of the features of front propagation into an unstable state is a special case of the Fisher-Kolmogorov (FK) equation, ${ }^{1,2}$

$$
\frac{\partial \phi}{\partial t}=\frac{\partial^{2} \phi}{\partial x^{2}}+\phi-\phi^{3} .
$$

Clearly, the state $\phi=0$ is unstable while the states $\phi= \pm 1$ are stable. The typical situation of interest is the one in which a front is moving to the right, replacing the unstable $\phi=0$ state by the stable $\phi=1$ state.

How fast will such a front move? The difficulty of answering this question becomes clear by considering the equation for the uniformly translating profile $\phi(x-v t)$, 
moving with a constant velocity $v$,

$$
-v \frac{d \phi}{d x}=\frac{d^{2} \phi}{d x^{2}}+\phi-\phi^{3},
$$

or, equivalently,

$$
\frac{d^{2} \phi}{d x^{2}}=-v \frac{d \phi}{d x}-\frac{d}{d \phi}\left(\frac{1}{2} \phi^{2}-\frac{1}{4} \phi^{4}\right)
$$

where the boundary conditions dictated by the problem are $\phi \rightarrow 0$ for $x \rightarrow \infty$ and $\phi \rightarrow 1$ for $x \rightarrow-\infty$. This equation has acceptable solutions for any value of the velocity $v$. To see this, we note that Eq. (1.2b) is equivalent to the equation of motion for a particle subject to friction moving in a potential $V=\frac{1}{2} \phi^{2}-\frac{1}{4} \phi^{4}$ with $x$ playing the role of time and $v$ the role of the friction constant. The kind of solution we are interested in is the one where the particle starts at the top of the potential $(\phi=1)$ at "time" $x \rightarrow-\infty$ and comes to rest in the bottom of the potential for long times $(x \rightarrow \infty)$ (see Fig. 1). Physically, it is clear that such a solution exists for any value of the "friction" $v$. In other words, steady-state fronts exist for any velocity $v$, so that the front propagation problem for Eq. (1.1) cannot be determined on the basis of steadystate considerations alone: Some sort of dynamical velocity selection must take place if under most conditions only one particular front speed is observed.

The mathematical analysis of Eq. (1.1) and the generalization

$$
\frac{\partial \phi}{\partial t}=\frac{\partial^{2} \phi}{\partial x^{2}}+F(\phi), \quad F^{\prime}(0)=1,
$$

culminated in the classical work by Aronson and Weinberger, published in $1975 .^{22}$ The conclusion of this study is that for a large class of functions $F(\phi)$ and for most "natural" initial conditions [those which are sufficiently localized so that $\phi(x, t=0)$ decays faster than $e^{-x}$ for large $x]$, the front velocity asymptotically ${ }^{23}$ approaches the value $v^{*}=2$. Similar type of results have also been derived for certain difference equations ${ }^{24}$ and an integral equation. $^{25}$

In spite of the mathematical progress made, a clear intuitive understanding of the reason that the velocity of typical fronts propagating into an unstable state quickly

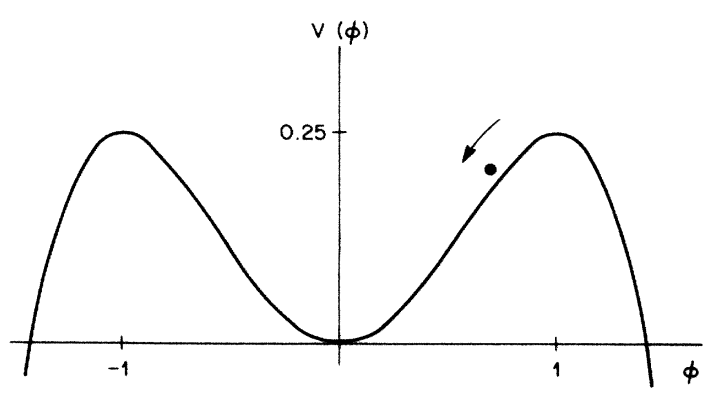

FIG. 1. Potential $V(\phi)=\frac{1}{2} \phi^{2}-\frac{1}{4} \phi^{4}$. Equation (1.2b) for a profile moving with steady-state velocity $v$ can be interpreted as the equation for a particle subject to friction moving down the potential. approach some asymptotic value $v^{*}$, and a simple method for calculating $v^{*}$, are still sought after. In 1983, however, an important step towards achieving this goal was made by Dee et al. ${ }^{5,6,8}$ They emphasized that the velocity $v^{*}$ for Eqs. (1.1) and (1.3) is just the one at which the front appears to be "marginally stable,", in that the front solutions that move slower than $v^{*}$ are unstable to perturbations (in the co-moving frame) while those that move faster are stable. ${ }^{27}$ If there is indeed an underlying connection between the existence of $v^{*}$ and marginal stability, this has great conceptual and practical implications, since the velocity corresponding to marginal stability can be determined explicitly from a simple analysis in the leading edge of the profile. ${ }^{28}$ In this region where $\phi$ is growing but still small, $\phi$ is approximately a solution of the linearized equation, and so

$$
\phi \sim e^{\omega t-k z},
$$

with $\omega(k)$ given by the dispersion relation of the linearized equation. When $\omega$ and $k$ are complex, this solution represents a front whose envelope moves with a velocity $v_{\text {env }}=\operatorname{Re} \omega(k) / \operatorname{Re} k$. For the leading edge where (1.4) holds, stability with respect to small changes in the wave vector $\operatorname{Im} k$ requires that $\operatorname{Im}(d \omega / d k)=0$. If this condition holds, the particular value of the envelope velocity where perturbations move with the profile must, as will be explained later, be equal to the group velocity $d \omega / d k$, so that $v_{\text {env }}=d \omega(k) / d k$. Then this profile is marginally stable. Hence if the selected velocity $v^{*}$ does indeed correspond to the marginal stability velocity, we have

$$
v^{*}=\frac{\operatorname{Re} \omega\left(k^{*}\right)}{\operatorname{Re} k^{*}}, \quad v^{*}=\left.\frac{d \omega(k)}{d k}\right|_{k^{*}},\left.\operatorname{Im} \frac{d \omega}{d k}\right|_{k^{*}}=0 \text {. }
$$

Since $\omega(k)$ is known for a given equation, these equations directly determine $k^{*}$ and $\omega\left(k^{*}\right)$, and hence $v^{*}$. For example, using that $\omega(k)=1+k^{2}$ for Eq. (1.1), one easily finds $v^{*}=2$, confirming the observation by Dee et al. ${ }^{5,6}$ that the profile corresponding to the selected speed $v^{*}$ turns out to be at the marginal stability point.

Dee et al. ${ }^{5,6,29}$ have also tested the validity of Eq. (1.5) numerically for front propagation in several other model equations, one of which is the Swift-Hohenberg equation $^{30}$ for real $\phi$,

$$
\frac{\partial \phi}{\partial t}=-2 \frac{\partial^{2} \phi}{\partial x^{2}}-\frac{\partial^{4} \phi}{\partial x^{4}}+(\epsilon-1) \phi-\phi^{3}, \epsilon>0 .
$$

For this equation, the patterns emerging behind the front are periodic in space. The numerical studies indicated that the selected velocity of the envelope of the front was always related to marginal stability via Eq. (1.5). In view of the difference in structure of Eq. (1.1) and the Swift-Hohenberg equation, ${ }^{31}$ as well as the difference between uniformly translating fronts relevant for Eq. (1.1) and those whose envelope is moving uniformly, these observations suggest that for this type ${ }^{32}$ of front propagation, some common dynamical mechanism is able to drive the front velocity to the marginal stability value. 
It is the purpose of this paper to identify this mechanism precisely for a wide class of one-dimensional equations. In particular, we will first present a simple intuitive explanation for this dynamical behavior, and then support our picture by a more mathematical analysis in which we associate Eq. (1.5) with a fixed point of a differential equation governing the dynamical evolution of the leading edge of the profile. The stability of this fixed point can be checked explicitly for a given equation. If the marginal stability point is stable, the naturally selected speed for sufficiently localized initial conditions will be $v^{*}$ as given by Eq. (1.5). As an example, we show that the fixed point for the Swift-Hohenberg equation (1.6) is stable, thus providing an after the fact justification for the numerical findings by Dee et al. ${ }^{5,6}$ We will also discuss an extension of the FK equation (1.1) for which, for a range of parameters, the dynamics of fronts is predicted to show behavior distinctly different from that arising in the FK equation.

Our analysis was inspired by and builds directly on recent work by Shraiman and Bensimon, ${ }^{33}$ who discussed the present velocity-selection problem for first-order partial differential equations. First-order equations are very helpful in understanding the dynamical mechanism that leads to marginal stability, but at the same time they lack most of the instabilities that can render the mechanism inoperative in higher-order partial differential equations. The work by Shraiman and Bensimon, ${ }^{33}$ in turn, has some elements in common with other formulations, ${ }^{34}$ but we have not made a complete comparison with all other methods in the extensive literature, which encompasses such diverse fields as biology, chemistry, applied mathematics, and physics. Nevertheless, we believe the present formulation, taken as a whole, to be largely new and to have the advantage that it focuses directly on the connection between velocity selection and marginal stability, and the way in which that connection results from some general properties of front propagation into an unstable state.

As mentioned earlier, propagating fronts can appear in several different forms. The simplest type of fronts are those whose long-time dynamics corresponds to a uniform translation of the profile as a whole, so that the solution becomes of the form $\phi(x-v t)$. We will refer to these as uniformly translating fronts, and they typically occur when the state emerging behind the front is homogeneous. Fronts in the FK equation are of this type and they do indeed lead to a homogeneous state $\phi=1$ or -1 behind the front. When the stable states of the system are periodic in space, however, uniformly translating fronts cannot occur, and we have to investigate the envelope of the front. We will therefore refer to these fronts as envelope fronts; these are the ones that induce dynamical pattern selection.

The fact that we will concentrate our analysis on the behavior of the leading edge of the profile where the dynamics are governed by the linearized equation, has, of course, its strength as well as its weakness. The power of the approach in this paper is that it leads to a simple picture and that it can explicitly predict the selected front velocity via Eq. (1.5) (provided the fixed point cor- responding to this equation is stable). The weakest point of our method is that it does not address the effects of the nonlinear terms on the dynamics, and that it makes no predictions if the marginal stability fixed point is not attractive. The effect of nonlinearities can, of course, only be analyzed for a specific equation. However, an example to be discussed later indicates that the most serious effect of nonlinearities on the marginal stability result is found when the profile behind the leading edge is subject to a strong nonlinear competition between a multiple set of stable steady states that can emerge behind the front. In the example, this competition only affects the structure of the state behind the front but not the validity of the marginal stability predictions for the velocity. Thus, provided they are used with some caution concerning such possibilities, it appears that our results generally provide a fairly complete description of the front dynamics.

The outline of this paper is as follows. In Sec. II we give a physical example of the type of front propagation we consider, and briefly touch on some of the differences between solitons and front propagation. We then exploit some analogies with well-known crystal-growth phenomena to arrive in an intuitive way at our main results. This is followed by the mathematical analysis in Sec. III, in which we associate Eq. (1.5) with a fixed point of the equation for the front profile and show how to check its stability (some of the mathematical questions underlying this approach are discussed in two appendixes). The effect of nonlinearities as well as some of the other open questions concerning our analysis are the subject of Sec. IV, while we summarize our main conclusions in Sec. V.

\section{PHYSICAL PICTURE}

In this section we give a descriptive discussion of the physics of front propagation into an unstable state in which we try to couch the main results from the literature and of Sec. III in an intuitive language. We start out by emphasizing the main differences with soliton motion, and then use a (hypothetical) example of wall motion in liquid crystals to illuminate the main results of the more detailed analysis.

In magnetic materials, walls or wall-like excitations generally move essentially without damping, as a result of which their dynamics are often well described by nonlinear wave equations that admit soliton solutions. For example, the continuum equation for a ferromagnetic chain with planar anisotropy and a symmetry-breaking field in this plane is described ${ }^{35}$ by the sine-Gordon equation $\phi_{t t}-c^{2} \phi_{x x}=m^{2} \sin \phi$, where $\phi$ is the angle between the spin and the field and where $\phi_{x}=\partial \phi / \partial x$, etc. Let us write this equation with a phenomenological damping term $\gamma \phi_{t}$ included, ${ }^{36}$

$$
\frac{\partial^{2} \phi}{\partial t^{2}}+\gamma \frac{\partial \phi}{\partial t}-c^{2} \frac{\partial^{2} \phi}{\partial x^{2}}=m^{2} \sin \phi,
$$

so that magnetic walls are described by this equation in the frictionless limit $\gamma \rightarrow 0$. In the absence of friction, walls (solitons) can move with a whole range of velocities $v$, and consequently the low-temperature spin dynamics 
of such chains can be understood in terms of a dilute gas of solitons with a distribution of velocities. ${ }^{36}$

In this paper, on the other hand, we are interested in wall or front propagation in systems with large damping $\gamma$, so that the term $\phi_{t t}$ in (2.1) can be neglected in comparison with the term $\gamma \phi_{t},{ }^{37}$ so that Eq. (2.1) becomes of the form (1.3).

In comparison with soliton systems $(\gamma \rightarrow 0)$, we physically expect the following qualitative behavior in the large damping regime.

(i) With large friction, freely propagating wall-like solutions cannot persist - they can only be temporarily created by suddenly quenching the system into an unstable state, as described in the introduction, and they do not have soliton character. ${ }^{38}$

(ii) One intuitively expects any front to quickly reach some asymptotic speed $v^{*}$, independent of the details of the initial conditions, since this is typical for systems with large dissipation.

These points are illustrated in Fig. 2 for the case of a wall in a nematic liquid crystal ${ }^{39}$ (this hypothetical case only serves to guide our intuition; see Refs. 10-12 for
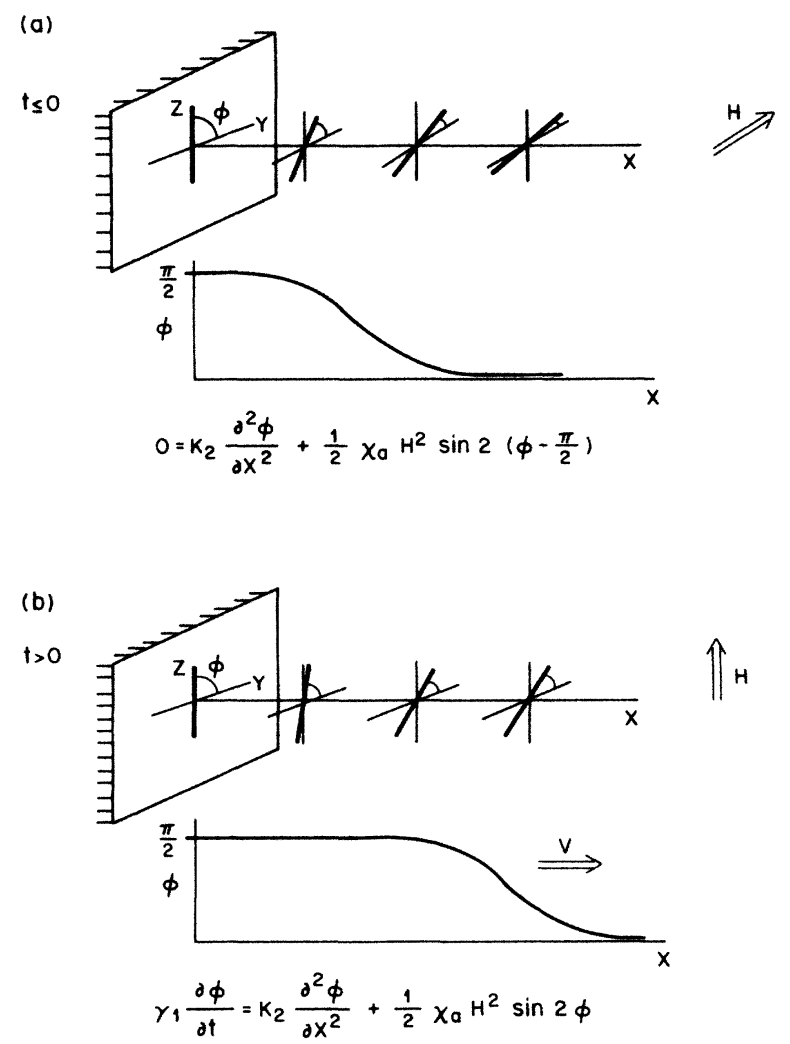

FIG. 2. Wall motion in a nematic liquid crystal induced by turning the magnetic field $\mathbf{H}$ through $90^{\circ}$. (a) For $\tau \leq 0$ the field points in the $y$ direction, while the director, indicated by a bold line, is anchored in the $z$ direction at the cell boundary. In the equation for the stationary profile $\phi(x), K_{2}$ is the twist elastic constant and $\chi_{a}$ the susceptibility (Ref. 39). (b) For $\tau>0$, the field points in the $z$ direction. Since the director at $x=0$ was already pointing in the $z$ direction at time $\tau=0$, the region where $\phi=\pi / 2$ expands so that a wall moves in the positive $x$ direction. In the equation, $\gamma_{1}$ is a twist viscosity. real experiments on liquid crystals). In nematics, the director can be oriented with a magnetic field $\mathbf{H}$. In the situation sketched in Fig. 2(a), the director is supposed to be anchored in the $z$ direction at the cell boundaries. Initially, the field $\mathbf{H}$ is in the $\boldsymbol{y}$ direction, and away from the cell boundary this causes the director to rotate to the $y$ direction [Fig. 2(a)]. At $t=0$, the field $\mathbf{H}$ is flipped to the $z$ direction. The state $\phi=0$ in the bulk is now unstable, and a front $\phi(x, t)$ starts to move in from the cell wall, as sketched in Figs. 2(b). Its velocity will be determined by the balance of the rate of change in the elastic and magnetic energy with the viscous dissipation. However, the wall width $W$ is not prescribed, and by allowing $W$ to vary one obtains a continuous range of solution, parametrized by $W$ or, equivalently, the velocity $v$.

If the equation would only admit steady-state propagation at one or a few values of the velocity $v$, these values would depend on all details of the equation, including the nonlinear terms. However, since a steadystate solution exist for a whole continuous range of values of $v,{ }^{40}$ the asymptotic velocity $v^{*}$ is essentially determined by a simple dynamical mechanism, well known in the theory of crystal growth. ${ }^{41}$ Consider the growth of the crystal of Fig. 3(a), whose initial shape is drawn by a solid line. The growth rate of the $A$ facets is slower than that of the $B$ facets. The successive stages during the growth of the crystal, drawn with dashed lines, clearly show that the fast-growing $B$ facets quickly become less important and may actually disappear completely: the asymptotic growth rate is dominated by that of the slowest facet, because the fast facets make themselves disappear (the same effect occurs if the crystal is not faceted but rough ${ }^{42}$ ). Essentially the same effect occurs for the type of front propagation discussed here, as is illustrated in Fig. 3(b). The profile in this figure consists of two parts that propagate with different speeds, as indicated by the arrows. Clearly, the slowest moving part (solid line) expands at the expense of the faster part (dashed line), and increasingly dominates the appearance of the profile, whose width is effectively seen to decrease in time. This dynamical effect is indeed the result of the fact that the velocity of a profile (or a portion thereof) increases with the width of the profile, which, as is illustrated in Fig. 3(c), is a general feature for fronts propagating into an unstable state. Although the discontinuities in the slope of Fig. 3(b) do not occur for the smooth profiles relevant for the equations we will study, ${ }^{43}$ essentially the same dynamical mechanism often turns out to exist there: the dynamical effect of fast portions is to squeeze the width of the profile, and this causes a decrease in speed.

In general, the steady-state profiles, parametrized by their velocity $v$, will be unstable for certain ranges of $v$ [see Fig. 3(d)]. In particular, because the propagation is into an unstable state, the profiles are expected to be unstable for small enough velocity, as indicated in Fig. 3(d). Naturally, the profile corresponding to the asymptotic speed cannot be unstable. This, together with the dynamic mechanism illustrated above and in Figs. $3(a)-3(c)$, suggests that the front speed will in general continue to slow down towards the smallest velocity in 
(a)

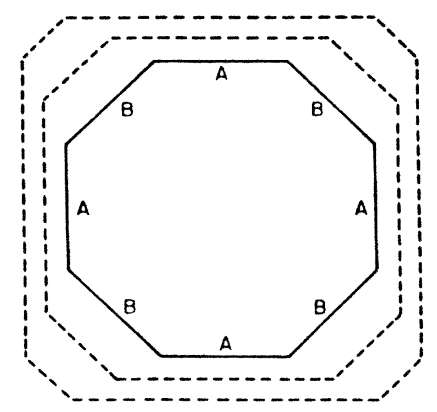

(b)

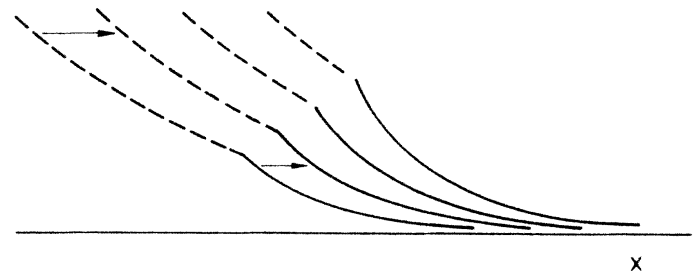

(c)

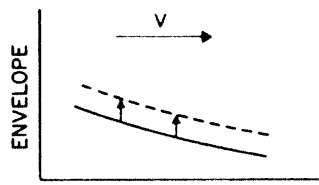

(d)

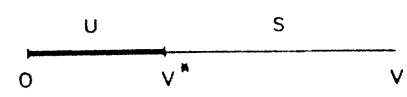

FIG. 3. (a) In crystal growth, it is well known that the growth of a crystal is dominated by the growth of the slowest facet, $A$ in this case. This can be viewed as a kind of velocity selection. In this figure, the dashed lines indicate two successive stages during growth of the crystal whose initial shape is drawn with a solid line. (b) Effects similar to those known for crystal growth can occur for a moving profile in the kind of equation discussed in this paper. The lower part of the profile, drawn with a solid line, moves slower than the dashed part. The figure illustrates how the crossover point moves up in time, so that the profile becomes more and more dominated by the slowly moving part. The fast part essentially works itself out of the leading edge. (c) Illustration of the fact that the velocity decreases with the steepness of the profile. When the two profiles grow about equally fast at fixed positions, as indicated by the vertical arrows, the envelope of the one with the smaller slope moves faster to the right. (d) When there is a continuum of steady-state profiles, parametrized by their velocity $v$, we in general expect the profiles for small $v$ to be unstable. $v^{*}$ is the velocity at which the stability changes, i.e., the marginal stability point.

the stable range. But this is precisely the velocity at which the stability of the profile changes; hence, according to this argument the front speed is expected to approach the velocity corresponding to the marginal stability point.

Let us now discuss why this marginal stability point is generally given by Eq. (1.5) (see also Refs. 5 and 6). The stability of the profile depends on the growth of perturbation as well as their propagation relative to the mov- ing profile. Consider first the growth. If we consider a profile $\phi(x, t) \sim \exp \left[-k_{0} x+\omega\left(k_{0}\right) t\right]$ ( $\omega$ and $k_{0}$ in general complex), it will be unstable against a perturbation with a slightly different wave vector $\left(k=k_{0}-i \epsilon\right.$ with $\epsilon$ real) when $\operatorname{Im}(d \omega / d k) \neq 0$, since then $\exp [\operatorname{Im}(d \omega / d k) \epsilon t]$ always diverges for values of $\epsilon$ of the same sign as Im $d \omega / d k$. Hence, front solutions that are stable in the frame moving with the profile must at least satisfy Im $d \omega / d k=0 .{ }^{44}$ This confirms the physical expectation that a profile will only be stable to small perturbations in the wave number $\operatorname{Im} k$ if $\operatorname{Im} k$ corresponds to the fastestgrowing mode (for fixed Rek). Clearly, $\operatorname{Im}(d \omega / d k)=0$ is a necessary, but not sufficient, condition for this. Next, consider the effect of propagation of perturbations on the stability. For the fronts which are not unstable against the above instability, and so for which Im $d \omega / d k=0$, a small localized disturbance or perturbation of the profile propagates, just as for simple Fourier waves, ${ }^{45}$ with the group velocity $v_{g}(k)=d \omega / d k$. Thus, if $v_{g}$ is larger than the envelope velocity $v_{\text {env }}=\operatorname{Re} \omega(k) /$ Rek with which the profile propagates, a small perturbation will move ahead relative to the front and cause the profile to be unstable. Similarly, profiles are stable if $v_{g}<v_{\text {env }}$, since then a perturbation disappears from the leading edge in much the same way as the breakpoint in Fig. 3(b) does. Consequently, the point where the fronts change stability with respect to this effect is the point at which $v_{\text {env }}=v_{g}$, i.e., $\operatorname{Re} \omega(k) / \operatorname{Re} k=d \omega(k) / d k$. Upon identifying the selected speed $v^{*}$ with the velocity at this point, as argued above, we recover Eq. (1.5). Note also that on the stable branch the group velocity $d \omega / d k$ (with which a small perturbation propagates) is less than the envelope velocity, just as the velocity of the breaking point in Fig. 3(b) is less than that of the solid curve. We will show in Sec. III that as a result the velocity of the profile is indeed driven towards the marginal stability value, provided no other instabilities intervene.

If Eq. (1.5) has multiple solutions, the above discussion suggests that we should associate the asymptotic front speed with the smallest of these. This is indeed correct for first-order partial differential equations, ${ }^{33}$ and probably also for fronts leading to periodic states. As we will discuss in Sec. IV, however, there is reason to believe that for uniformly moving fronts $\phi(x-v t)$ of higher-order partial differential equations, only the solution corresponding to the smallest value of $\operatorname{Re}^{*}$ is relevant for the asymptotic behavior of the profile. If so, the asymptotic speed of these uniformly moving fronts will be the $v^{*}$ corresponding to the smallest value of $\operatorname{Re}^{*}$.

We stress again that even though the picture presented here captures most of the essential physics, marginal stability only applies under more restricted conditions. A simplified form of the additional condition follows directly from the earlier discussion; clearly a profile $\exp [-k x+\omega(k) t]$ satisfying (1.5) will only be stable to perturbations $i \delta k^{i}$ in $k$ if the growth rate $\omega(k)$ is maximal, so that $\operatorname{Re}^{2} \omega / d k^{2}\left(i \delta k^{i}\right)^{2}<0$. Thus a necessary additional condition for stability of front solutions is

$$
\operatorname{Re} \frac{d^{2} \omega}{d k^{2}}>0 \text {. }
$$


The generalization of this condition, which already has important implications for uniformly translating fronts, will be discussed in Sec. III C.

In discussing Fig. 3, we have not yet paid attention to the effect of initial conditions. Let us first return to the physical analogy of Fig. 3(a). If the crystal has no $A$ facets initially, and if these facets cannot nucleate, then the crystal would actually grow with the higher growth rate of the $B$ facets. Similarly, in front propagation it is essential that the initial profile drops off fast enough. ${ }^{22,33}$ That is, if we consider instead of Fig. 3(b) a profile where the asymptotic (large $x$ ) behavior is given by the fastmoving dashed portion, this portion actually expands in time and therefore dominates the long-time behavior. In other words, the slowing down of the speed and approach of the marginal stability value only occurs if the faster moving parts "catch up" with the slower parts. This is the case when the initial conditions are sufficiently localized. On the other hand, if the initial conditions are such that $\phi(x, t=0)$ drops off slower in $x$ than the profile corresponding to the marginal stability point $\left[\phi \sim \exp \left(-\operatorname{Re}^{*} x\right)\right]$, it is found that the front can move with a higher speed. Whether such exceptions are in fact relevant in practice, however, also depends on the importance of fluctuations, since we expect the deviations from the marginal stability value due to initial conditions to show up only in the region where the initial $\phi(x, t=0)$ is larger than the typical fluctuating value in the unstable state. ${ }^{46}$ Also, new walls nucleated in the bulk would presumably propagate with $v^{*}$.

In the "experiment" of Figs. 2(a) and 2(b) $\phi(x, t=0)$ falls off exponentially with a decay length inversely proportional to $H_{i}$, the field strength before switching. According to the theory, the front speed is therefore determined by the initial conditions in the regime $H_{i}<H_{f}$, where $H_{f}$ is the field after switching; the predicted velocity in this regime is ${ }^{47}$

$$
v=\left(\chi_{a} K_{2} / \gamma^{2}\right)^{1 / 2} H_{i}\left(1+H_{f}^{2} / H_{i}^{2}\right)
$$

while for $H_{i} \geq H_{f}$ one should have the marginal stability result $v=2\left(\chi_{a} K_{2} / \gamma^{2}\right)^{1 / 2} H_{f}$. It would be interesting if a real experiment of this type could be done, since this would test the relevancy of the initial condition in a physical system with fluctuations.

\section{MATHEMATICAL ANALYSIS OF THE DYNAMICS IN THE LEADING EDGE}

\section{A. Introduction and notation}

In this section we extend the approach of Shraiman and Bensimon ${ }^{33}$ to arbitrary partial differential equations in one dimension which are of first order in time but of arbitrary order in the spatial derivatives, and whose $\phi=0$ state is linearly unstable. While our analysis is quite general, it will often be helpful to illustrate the discussion by considering specific examples such as the complex generalization of Eq. (1.1), the "amplitude equation" 7,8,48 (AE) for complex $\phi$,

$$
\frac{\partial \phi}{\partial t}=\frac{\partial^{2} \phi}{\partial x^{2}}+\phi-|\phi|^{2} \phi,
$$

as well as the Swift-Hohenberg (SH) equation (1.6). Like the latter, Eq. (3.1) allows periodic steady states, and it was therefore also studied in the context of front propagation by Dee et al. ${ }^{6,8}$ Of course, a number of aspects of the analysis for (3.1) is the same as for the FK equations. Nevertheless, we prefer to use the AE as an example to stress that most of our arguments apply to uniformly translating fronts as well as to envelope fronts. As a third example we will consider the equation obtained by adding a stabilizing fourth-order derivative to Eq. (1.1):

$$
\frac{\partial \phi}{\partial t}=\frac{\partial^{2} \phi}{\partial x^{2}}-\gamma \frac{\partial^{4} \phi}{\partial x^{4}}+\phi-\phi^{3}
$$

We will refer to this as the extended FK (EFK) equation. To our knowledge, this equation has not been studied before from the point of view of front propagation. In contrast to the SH equation, where there is a finite wavelength instability due to the different sign of the second derivative term, all spatial derivatives in the EFK equation are stabilizing, and we intuitively expect this equation to have solutions of the type found in Eq. (1.1), for which the $\phi=1$ state emerges behind the front.

We will assume that the general type of equation we consider admits a continuous family of stable steadystate fronts, each advancing with a different speed $v$ (see Ref. 40 and in Appendix A for further discussion of this point). We argued physically in Sec. II that in such cases the velocity is expected to be determined by some dynamical mechanism. By focusing immediately on the dynamics in the leading edge of the front, we make the implicit assumption that the dynamics leading to selection in that region is representative for that in other parts of the profile as well. A detailed investigation of the validity of this assumption is beyond the scope of this paper, but such an assumption seems reasonable for most cases of interest, in which there are no particular pathologies that lead to additional (localized) instabilities behind the leading edge (for further discussion, see Sec. IV). In any case, it appears that the conditions we will derive are at least necessary conditions for the marginal stability result to be valid.

In the general case, the leading edge of the profile propagating into the unstable $\phi=0$ state will obey a linearized equation of the form ${ }^{49}$

$$
\frac{\partial \phi}{\partial t}=F\left(\phi, \phi_{x x}, \ldots\right)
$$

where $F$ is linear in $\phi, \phi_{x x}$, etc. For profiles of the form (1.4), this equation determines the dispersion relation 
$\omega(k)$, with $\omega$ and $k$ complex and $\omega(k)=F\left(1, k^{2}, \ldots\right)$. For the AE, and SH and EFK equation, respectively, we have, e.g.,

$$
\begin{aligned}
& \omega(k)=1+k^{2}, \\
& \omega(k)=\epsilon-1-2 k^{2}-k^{4}, \\
& \omega(k)=1+k^{2}-\gamma k^{4} .
\end{aligned}
$$

Since $\phi$ drops off approximately exponentially, it is advantageous to transform to the variable $u$ by writing $\phi=e^{-u}$; in doing so, we have to allow $u$ to be complex, since in the AE and SH equation $\phi$ is oscillatory in the leading edge. The condition that $\phi \rightarrow 0$ for $x \rightarrow \infty$ thus becomes $u^{r} \equiv \operatorname{Re} u \rightarrow \infty$ for $x \rightarrow \infty$ (henceforth superscripts $r$ and $i$ are used to denote the real and imaginary part of a complex quantity, while subscripts $t$ or $x$ denote differentiation with respect to $t$ and $x$ ). Upon substitution of this transformation into Eq. (3.3), the dynamical equation for $u$ becomes

$$
u_{t}=-f\left(q, q_{x}, q_{x x}, \ldots\right)
$$

where we defined

$$
q \equiv u_{x}
$$

so that $f$ contains spatial derivatives of $q$ up to order $n-1$, if $F$ in (3.3) contains spatial derivatives of $\phi$ up to order $n$. In general, $f$ will be nonlinear in $q$ and its derivatives; e.g., for the AE, the SH, and the EFK equation, respectively, we get

$$
\begin{aligned}
& f\left(q, q_{x}\right)=1-q_{x}+q^{2} \\
& \begin{aligned}
f\left(q, q_{x}, q_{x x}, q_{x x x}\right)= & \epsilon-1-2 q^{2}-q^{4}+2 q_{x}\left(1+3 q^{2}\right) \\
& -3 q_{x}^{2}-4 q q_{x x}+q_{x x x}
\end{aligned}
\end{aligned}
$$

$$
\begin{aligned}
& f\left(q, q_{x}, q_{x x}, q_{x x x}\right) \\
& \qquad \begin{aligned}
=1+q^{2}-q_{x}+\gamma( & -q^{4}+6 q^{2} q_{x} \\
& \left.-3 q_{x}^{2}-4 q q_{x x}+q_{x x x}\right) .
\end{aligned}
\end{aligned}
$$

Note that when $q(x)=k$, independent of $x$ [throughout this paper, $\omega$ and $k$ will always be (complex) numbers, not functions of $x$ ], we have

$$
f(k)=\omega(k),
$$

where $f(k)$ is shorthand for $f\left(q=k, q_{x}=0, q_{x x}\right.$ $=0, \ldots)=F\left(1, k^{2}, k^{4}, \ldots\right)$.

\section{B. Stability of front solutions}

\section{Stable solutions}

In a frame moving with a constant velocity $v$, we have

$$
u_{t}=v q-f\left(q, q_{x}, \ldots\right) \text {. }
$$

Consider now a steady-state front solution, i.e., a solution $q(x, t)=k$ (=const) whose envelope propagates with a constant speed $v$ (written as $v_{\text {env }}$ earlier) in the laboratory frame. For such a solution $\operatorname{Re} u_{t}=0$ in the moving frame (since we only require here the envelope to move with a constant speed), and hence we recover from Eq. (3.9) the result already discussed in Secs. I and II,

$$
\operatorname{Re}[v k-f(k)]=0 \rightarrow v(k)=\frac{\omega^{\prime}(k)}{k^{r}} .
$$

To study the stability of these solutions, we consider a small perturbation $\delta$ in $u$; upon linearizing (3.9) around the steady-state solution, we get

$$
\delta_{t}=\left[v(k)-f_{q}(k)\right] \delta_{x}-f_{q_{x}}(k) \delta_{x x}-\cdots,
$$

where $f_{q_{x}}(k)$ denotes $\partial f\left(q, q_{x}, \ldots\right) / \partial q_{x}$ evaluated at $q=k$, etc., and where $\cdots$ stands for terms involving higher-order derivatives of $\delta$. For the profile to be stable, bounded perturbations of the form $\delta \sim e^{-\mu x}$ with $\operatorname{Re} \mu>0$ need to decay, i.e., for these $\operatorname{Re}\left(\delta_{t} / \delta\right)<0$. For small $|\mu|$ only the first term in Eq. (3.11) contributes, and hence a necessary condition for stability is

$\operatorname{Re}\left\{-\left[v(k)-f_{q}(k)\right] \mu\right\}<0$ for all small $\mu, \operatorname{Re} \mu>0$.

This condition can only be satisfied if

$$
\begin{aligned}
& \operatorname{Im} f_{q}(k)=0, \rightarrow \operatorname{Im} \frac{d \omega(k)}{d k}=0, \\
& \operatorname{Re}\left[v(k)-f_{q}(k)\right]>0 \rightarrow v(k)>\operatorname{Re} \frac{d \omega(k)}{d k} .
\end{aligned}
$$

These are precisely the necessary conditions for stability discussed in Sec. II, where we obtained (3.14) by arguing that for a front to be stable its speed should be larger than the "group velocity" with which perturbations propagate, $v_{g}=d \omega / d k$ [provided $\operatorname{Im}(d \omega / d k)=0$ as required by (3.13); additional requirements such as (2.2) will be discussed later].

Any wave front can only be stable against sinusoidal perturbation at the values of $k$ where condition (3.13) is obeyed. Let us use this equation to express the imaginary part $k^{i}$ as a function of $k^{r}$, so that we can then obtain the velocity $v=\omega^{r} / k^{r}$ of these solutions as a function of $k^{r}$ as shown in Fig. 4(a); note that since we consider propagation into an unstable state, $\omega^{r}$ will approach a positive value for $k^{r} \rightarrow 0$. Hence $v$ generally diverges for $k^{r} \rightarrow 0$, as was already illustrated in Fig. 3(c). Such behavior is indeed found for the AE and SH equation, as depicted in Fig. 4(b) (the EFK equation will be discussed later). In order that the solutions depicted in Fig. 4 are also stable against the propagation of small 

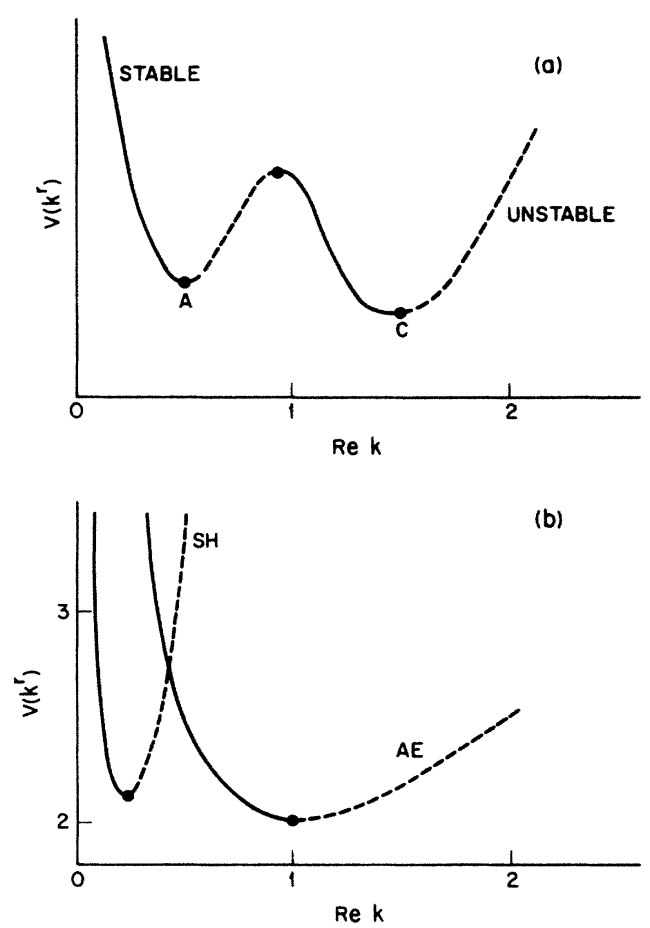

FIG. 4. (a) General behavior of $v$ as a function of Rek for solutions satisfying $\operatorname{Im} d \omega / d k=0$. The part of the curve drawn with thick solid lines denotes unstable branches. (b) $v$ as a function of $\operatorname{Re} k$ for the $\mathrm{AE}$ and the $\mathrm{SH}$ equation with $\epsilon=\frac{1}{4}$.

perturbations relative to the front, condition (3.14) has to be satisfied, too. Clearly, according to this condition the solutions are stable for small $k^{r}$ where $v$ diverges. To investigate where the stability changes, consider the extrema of the function $v\left(k^{r}\right)=\omega^{r} / k^{r}$, which satisfy ${ }^{50}$

$$
\begin{aligned}
\frac{d v\left(k^{r}\right)}{d k^{r}} & =\frac{1}{k^{r}}\left(-\frac{\omega^{r}}{k^{r}}+\frac{d \omega^{r}}{d k^{r}}\right) \\
& =-\frac{1}{k^{r}}\left[v\left(k^{r}\right)-\operatorname{Re} \frac{d \omega}{d k}\right)=0 .
\end{aligned}
$$

Comparison of this result with Eq. (3.14) shows that the stability of the solutions changes at the extrema of the function $v\left(k^{r)}\right.$; these extrema therefore coincide with the marginal stability points $v^{*}, k^{*}$ defined as the solutions of [cf. Eq. (1.5)]

$$
\operatorname{Im} \frac{d \omega}{d k}=0, v=\frac{\omega^{r}}{k^{r}}=\frac{d \omega}{d k} .
$$

Since the solutions are stable for $k^{r} \rightarrow 0$, we can immediately label the stability of various branches, as indicated in Fig. 4.

Two points should be stressed concerning the interpretation of the results depicted in Fig. 4. First of all, although we will for simplicity refer to the branch satisfying (3.12) as the stable branch, (3.12) only ensures the linear stability of the steady-state solution against perturbation with small $|\mu|$, not those with any $\mu$. For a particular equation, the stability analysis for finite $\mu$ is automatically included in the discussion of the behavior in the neighborhood of the marginal stability point, to be presented in Sec. III C.

Second, we note that in the linear analysis performed here, each value of $k^{r}$ is analyzed separately, assuming that this value represents the asymptotic decay of the profile. As Fig. 4(a) illustrates, in general several values of $k^{r}, k_{1}^{r}, k_{2}^{r}, \ldots$, say, may correspond to the same velocity. When there is a one-parameter family of front solutions, as in the case of the FK and EFK equation, only the smallest one of these, $k_{1}^{r}$, say, is expected to correspond to the actual asymptotic behavior of the profile. The counting argument discussed in Appendix A shows that this is the case in general for uniformly moving solutions, so that only the leftmost stable branch in the $v$ versus $k^{r}$ plot is relevant. As will be discussed further in Sec. IV, however, front propagation leading to periodic states is different; here all stable branches can in principle be relevant, because of an additional freedom present in this case (the wavelength of the periodic states behind the front).

\section{Unstable branches}

The above discussion of stable front solutions is quite general, and valid for uniformly translating fronts as well as for envelope fronts. To make contact with and extend earlier work, 6,22 we now also discuss some aspects of certain unstable front solutions. Unfortunately, this can only be done for uniformly translating profiles of the form $\phi(x-v t)$. In the leading edge, such solutions have to satisfy

$$
v k=\omega(k) \text {. }
$$

Instead of Eq. (3.10), we now have an equation for the real and imaginary part, and this equation can therefore again be used to express $v$ as a function of $k^{r}$ [stable uniformly translating steady-state solutions are, of course, also contained in (3.17)]. For example, for (1.1) or the amplitude equation, $\omega(k)=1+k^{2}$, so that (3.17) yields $k=\frac{1}{2}\left[v \pm\left(v^{2}-4\right)^{1 / 2}\right]$. For $v>2, k$ is real and we recover the stable solutions discussed before. For $v<2$, we have unstable solution [since Im $d \omega / d k=2 k^{i} \neq 0$, cf. Eq. (3.13)], with $v=2 k^{r}$. This branch of unstable solutions is indicated by a dotted line in Fig. 5(a). Note that the stable and unstable branches meet at the marginal stability point $k^{r}=1, v=2$. This typically occurs in a range of parameter values for equations that admit uniformly translating solutions, and whose $\omega(k)$ is real for real $k,{ }^{51}$ as we now proceed to show.

When $\omega(k)$ is real for real $k$, Eq. (3.13) has the trivial solution $k^{i}=0$, so that the stable branch will generally correspond to the one where $k^{i}=0, v\left(k^{r}\right)=\omega\left(k^{r}\right) / k^{r}$. Now consider the intersection of this branch with an (unstable) branch of solutions along which $k^{i}$ vanishes when approaching the intersection. In this neighborhood of the stable branch where $\partial \omega^{r} / \partial k^{i}=-\partial \omega^{i} / \partial k^{r}$ $=0$, we have for small $k^{i}, \delta v$, and $\delta k^{r}$,

$$
(v+\delta v) k^{i}=\operatorname{Re}\left(\frac{d \omega}{d k}\right) k^{i}+\frac{1}{2} \operatorname{Im}\left(\frac{d^{2} \omega}{d k^{2}}(\delta k)^{2}\right),
$$



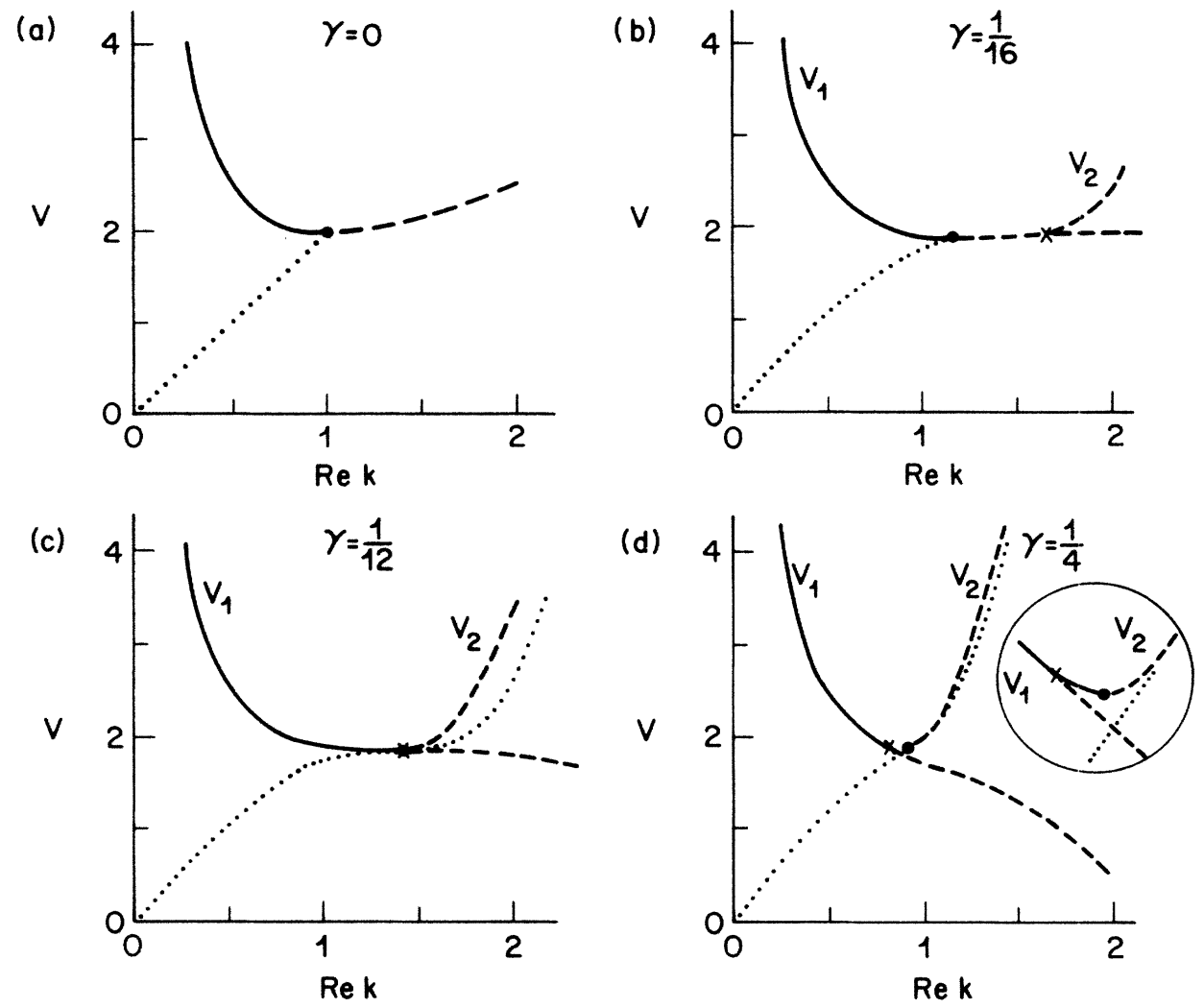

FIG. 5. $v$ vs Rek behavior for the EFK equation of four different values of $\gamma$. The solid line indicates the stable branch obeying (3.13) and (3.14) and the dashed line the extension of this branch to points where (3.13) holds but where (3.14) is violated, or where (2.2) or its generalization discussion in Sec. III C shows that the profile becomes unstable. This instability sets in along the branch labeled $v_{1}$ at the point indicated by a cross, and at this point also a new branch $v_{2}$ bifurcates off. The dotted line denotes a branch of uniformly moving solutions that are unstable because $\operatorname{Im} d \omega / d k \neq 0$, and the dot the marginal stability point. (a) $\gamma=0$; in this case the equation is equivalent to the AE. (b) $\gamma=\frac{1}{16}$; (c) $\gamma=\frac{1}{12}$, the largest value at which a marginal stability point lies on the branch $v_{1}$; (d) the situation for $\gamma=\frac{1}{4}$ sketched here is typical for all $\gamma>\frac{1}{12}$. The inset gives a detailed view of the region where the various branches intersect.

$$
\begin{aligned}
& \delta v \delta k^{r}+v \delta k^{r}+k^{r} \delta v \\
& =\operatorname{Re}\left(\frac{d \omega}{d k}\right) \delta k^{r}+\frac{1}{2} \operatorname{Re}\left(\frac{d^{2} \omega}{d k^{2}}(\delta k)^{2}\right) .
\end{aligned}
$$

Since (3.18a) contains a term linear in $k^{i}$, and since $k^{i} \sim\left(\delta k^{r}\right)^{2}$ is excluded if the two branches intersect at a finite angle, Eq. (3.18a) can only be obeyed if the terms linear in $k^{i}$ cancel, i.e., if the two lines intersect at the marginal stability point, where $v=\operatorname{Re}(d \omega / d k)$. At this point we then find from Eqs. (3.18a) and (3.18b) $\delta v=\operatorname{Re}\left(d^{2} \omega / d k^{2}\right) \delta k^{r}, \quad\left(\operatorname{Re} d^{2} \omega / d k^{2}\right)\left(\delta k^{i}\right)^{2}=-2 k^{r} \delta v$, which explicitly shows that if a marginal stability point exists, an unstable branch ends at this point, too. ${ }^{52}$ The only way in which an unstable branch can intersect the stable one is if $k^{i}$ does not vanish upon approaching the intersection along the unstable branch. This is possible if the equation $\omega\left(k^{r}, 0\right)=\omega\left(k^{r}, k^{i}\right)$ has a solution for some nonzero $k^{i}$.

These conclusions are nicely illustrated by an analysis of the EFK equation. For $\gamma=0$, this equation reduces to the AE equation, whose diagram is shown in Fig. 5(a). For small $\gamma>0$, the curves are shifted, but as predicted above, they still intersect at the marginal stability point, see Fig. 5(b) (explicit expressions are given in Appendix B). Moreover, Fig. 5(b) also shows that for $\gamma>0$ an additional branch of solutions, labeled $v_{2}$, arises. When $\gamma=\frac{1}{12}$, Fig. 5(c) shows that all branches intersect at an inflection point, and as a result for $\gamma$ larger than this value [Fig. $5(\mathrm{~d})$ ] the marginal stability point has moved onto the $v_{2}$ branch and the stable and unstable branches intersect at some arbitrary value. We will discuss Fig. 5(d) and its implications later.

\section{Dynamics in the leading edge}

We will now discuss, following the ideas of Shraiman and Bensimon, ${ }^{33}$ how and under what conditions the dynamics in the leading edge drives the front velocity to the marginal stability value.

The basic dynamical equation, Eq. (3.5), reads $u_{t}=-f\left(q, q_{x}, q_{x x}, \ldots\right)$. Let us restrict ourselves to profiles whose envelope is monotonically decreasing, so that $u^{r}(=\operatorname{Re} u)$ is a monotonically increasing function of $x$. It is then advantageous to write an equation for the evolution of $q$ (whose real part plays the role of a local 
inverse decay length for the profile and its imaginary part that of a wave vector) in terms of the variables $u^{r}$ and $t$ rather than $x$ and $t$, since the real part of $u$ moves with the (envelope of the) profile. ${ }^{53}$ Using

$$
\begin{aligned}
& \left.\frac{\partial}{\partial z}\right|_{t}=\left.q^{r} \frac{\partial}{\partial u^{r}}\right|_{t}, \\
& \left.\frac{\partial}{\partial t}\right|_{x}=\left.\frac{\partial}{\partial t}\right|_{u}+\left.\left.\frac{\partial u^{r}}{\partial t}\right|_{x} \frac{\partial}{\partial u^{r}}\right|_{t}=\left.\frac{\partial}{\partial t}\right|_{u}-\left.f^{r} \frac{\partial}{\partial u^{r}}\right|_{t}
\end{aligned}
$$

we get

$$
\begin{aligned}
\frac{\partial}{\partial u^{r}} \frac{\partial u(x, t)}{\partial t} & =\frac{1}{q^{r}} \frac{\partial^{2} u(x, t)}{\partial t \partial x} \\
& =\left.\frac{1}{q^{r}} \frac{\partial q}{\partial t}\right|_{x}=\frac{1}{q^{r}}\left(\left.\frac{\partial q}{\partial t}\right|_{u}-f^{r} q_{u}\right) .
\end{aligned}
$$

Here the subscript $u$ denotes a differentiation with respect to $u^{r}$, and $\left.\right|_{u}$ that the differentiation is performed with $u^{r}$ held constant. Differentiation of Eq. (3.5) with respect to $u^{r}$ then yields together with this result

$$
q_{t}=\left(\frac{f^{r}}{q^{r}}-f_{q}\right) q^{r} q_{u}+\mathcal{L} q,
$$

with

$$
\begin{aligned}
\mathcal{L} q \equiv & -f_{q_{u}}\left(q, q_{u}, q_{u u}, \ldots\right) q_{u u} \\
& -f_{q_{u u}}\left(q, q_{u}, q_{u u}, \ldots\right) q_{u u u}-\cdots .
\end{aligned}
$$

We wish to understand from this equation how an initially nonuniform function $q(u, t)$ evolves in time and approaches a constant value everywhere. Although $q=k$ (=const) is a solution of Eq. (3.21), we recognize in the term between square brackets for $q=k$ the combination $v(k)-f_{q}$ that according to Eq. (3.14) determines the stability of solutions, and which is zero at the marginal stability point. Therefore, the marginal stability point corresponds to a special type of fixed point of this equation, and the relevant nonlinearities for velocity selection are in the first term on the right-hand side of this equation. To illustrate this, consider the case in which the highest derivative in $\mathcal{L}$ is of second order, as is the case for the AE. To a first approximation, we can neglect the dependence of derivatives of $f$ in (3.22) on $q, q_{u}$, etc., so that $\mathcal{L} q \simeq D q_{u u}$ with $D=-f_{q_{u}}$, and consider $q^{r}\left(f^{r} / q^{r}-f_{q}\right)$ as a function of $q$, say, $c(q)$, only [note that along the stable branch, $c(q)$ is real in view of Eq. (3.13)]. We then get

$$
\frac{\partial q}{\partial t}=c(q) \frac{\partial q}{\partial u^{r}}+D \frac{\partial^{2} q}{\left(\partial u^{r}\right)^{2}} \text {. }
$$

This equation is of the form of the well-known Burgers equation. 45,54 The effect of the nonlinear "convective" term $c(q) \partial q / \partial u^{r}$ in this equation is well understood, ${ }^{45,54}$ and it is easy to show that this term drives $q$ for sufficiently localized initial conditions towards the mar- ginal stability value $q=k^{*}$ at which $c(q)=0$. In the general case of Eq. (3.21) our strategy will therefore be to first investigate under which conditions the first term on the right-hand side forces $q$ to approach the marginal stability fixed point, and then to check, for each particular equation, that the eigenmodes of the operator $\mathcal{L}$ are stable at this point. If they are, we have shown that the marginal stability point is attractive so that $v \rightarrow v^{*}$ for certain initial conditions.

Let us therefore consider for the moment only the first term in Eq. (3.21), so that

$$
q_{t}=\left(f^{r}-q^{r} f_{q}\right) q_{u},
$$

where we take $f$ as a function of $q$ only. To study the (nonlinear) stability of the marginal stability fixed point, let us write $q\left(u^{r}, t\right)=k^{*}+\rho\left(u^{r}, t\right)$. To lowest nontrivial order in $\rho$, we then have

$$
\frac{\partial \rho}{\partial t}=-\left(f_{q q} k^{r}\right)^{*} \rho \rho_{u}, \quad\left(f_{q q} k^{r}\right)^{*} \text { positive . }
$$

The fact that the term in parentheses is positive for physically relevant solutions follows from the fact that the term in parentheses in (3.24) changes sign at $q=k^{*}$, and that this term was found to be positive for $q^{r}<k^{r}$ [indeed $\left(f_{q q} k^{r}\right)^{*}$ is equal to $\left(k^{r}\right)^{2} \partial^{2} v /\left.\left(\partial k^{r}\right)^{2}\right|_{k=k^{*}}$, which is positive].

Consider first the most important case in which the initial profile is sufficiently localized, i.e., drops off faster than $\exp \left[-\left(\operatorname{Re} k^{*}\right) x\right]$. In terms of $q^{r}$, this means that $q^{r}$ is larger than $\operatorname{Re} k^{*}$ for large $u^{r}$, as sketched in Fig. 6(a). Since $\rho_{u}$ is positive in this case, $\rho$ decays according to Eq. (3.25) and hence $q^{r}$ approaches $\operatorname{Re} k^{*}$ asymptotically for all $u^{r}$, and by implication the front velocity $v$ approaches $v^{* 55}$ This is, for general equations, the analog of the Aronson and Weinberger ${ }^{22}$ results for Eq. (1.1) that $v$ approaches the marginal stability value $v^{*}=2$ for sufficiently localized initial conditions.

In the case that the initial conditions fall off less fast than $\exp \left[-\left(\operatorname{Re} k^{*}\right) x\right], q^{r}$ is smaller than $\operatorname{Re} k^{*}$ for large $u^{r}$. For the cases depicted in Figs. 6(b) and 6(c), we see that the first term in (3.21) drives $q^{r}$ away from the marginal stability value and towards its large $u^{r}$ value which is smaller [as can be seen in the figure, $q^{r}$ in Fig. 6(c), initially develops a shocklike structure at the point where $q^{r}=\left(k^{r}\right)^{*}$; using an argument similar to that of Bensimon and Shraiman, ${ }^{33}$ It can be shown that this structure eventually moves away towards smaller values of $u^{r}$, just as we saw in Fig. 3(b)]. In view of our discussion of Sec. III B, the speed of the profile will in both cases approach a value larger than $v^{*}, 56$ determined by the asymptotic behavior of $\phi(x, t=0)$ (cf. the discussion in Sec. II and Ref. 47). Again, specialized to Eq. (1.1), these results agree with those obtained rigorously by Aronson and Weinberger. ${ }^{22}$

The above results concerning the approach of the marginal stability point are only valid provided the eigenmodes of the linear operator $\mathcal{L}$, obtained from $\mathcal{L}$ by evaluating $f_{q_{u}}$, etc., in (3.22) at $q=k$, are stable at the marginal stability point. This requirement is the generalization of condition (2.2) to arbitrary wavelength perturbations. For the terms appearing in $\mathcal{L}$, it is easy to 

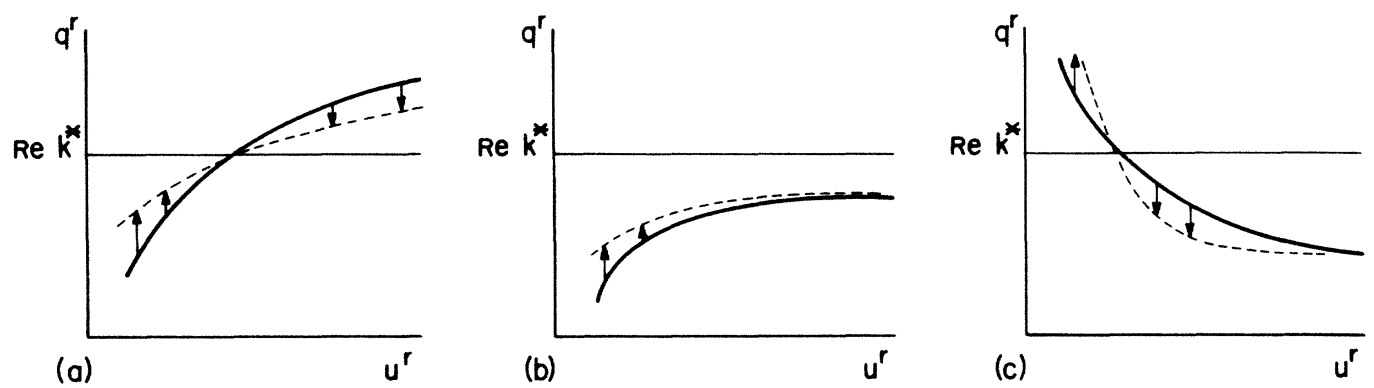

FIG. 6. Qualitative sketch of the dynamical behavior of the function $q^{r}$ for three different situations. The initial $q^{r}$ is drawn with a solid line and the one at a later time with a dashed line. (a) If the initial profile falls off faster than $\exp \left(-\operatorname{Re} k^{*} x\right), q^{r}$ approaches $\operatorname{Re} k^{*}$ in time and the velocity of the profile approaches $v^{*}$. (b) When $q^{r}$ tends to some value $q_{a s}^{r}<\operatorname{Re} k^{*}$ for large $u^{r}, q^{r}$ approaches $q_{a s}^{r}$ in time. (c) As in (b), $q_{a s}^{r}$ is approached for large $u^{r}$; initially, a shock region develops near the point where $q^{r}=\operatorname{Re} k^{*}$. This shock region later disappears to smaller values of $u^{r}$.

show that

$$
\begin{aligned}
& \left.f_{q_{u}}\left(q, q_{u}, q_{u u}, \ldots\right)\right|_{q=k}=\left.\operatorname{Re} k f_{q_{x}}\left(q, q_{x}, q_{x x}, \ldots\right)\right|_{q=k}, \\
& \begin{aligned}
&\left.f_{q_{u u}}\left(q, q_{u}, q_{u u}, \ldots\right)\right|_{q}=k \\
&=\left.(\operatorname{Re} k)^{2} f_{q_{x x}}\left(q, q_{x}, q_{x x}, \ldots\right)\right|_{q=k},
\end{aligned}
\end{aligned}
$$

so that $\mathcal{L}$ can be read off directly from the results given in Eqs. (3.7). We now check the stability for the three examples discussed before.

Example I: the amplitude equation. For this equation, we get according to Eqs. (3.7a), (3.22), and (3.26) for perturbation around $k^{*}=1$

$$
q_{t}=\mathcal{L} q=\left(k^{*}\right)^{2} q_{u u}=q_{u u},
$$

so that perturbations indeed decay in time. This finally establishes the stability of the marginal stability fixed point of the $\mathrm{AE}$ equation. We have thus rederived, within the context of our method, the results of Aronson and Weinberger for Eqs. (1.1) and (1.3), and at the same time generalized these results to the AE (3.1). The result $v^{*}=2$ is easily derived from Eqs. (1.5).

In passing, we note that in Sec. III B we have checked the stability of the "stable branch" only for longwavelength perturbations; however, if the linearized operator $\mathcal{L}$ is stable all along this stable branch, then this immediately implies the stability against finite wavelength perturbations of solutions on this branch, too. When linearizing Eq. (3.21) away from the marginal stability point, the first term on the right can be transformed away by going to a frame moving, according to (3.14), to smaller $u^{r}$ values, i.e., away from the leading edge. Together with the stability of the operator $\mathcal{L}$, this then implies the linear stability of the steadystate profile. For the $\mathrm{AE}$, this is indeed the case since $\mathcal{L} q=k^{r} q_{u u}$ along the stable branch. Note also that, as a result, the equation for $q_{t}$ along the stable branch is essentially the Burgers-like equation (3.23).

Example II: the $S H$ equation. For this equation, $\mathcal{L}$ can again be evaluated easily at any point along the stable branch. Upon substitution of $q=k+\exp (\Omega t$ $\left.+i Q u^{r} / k^{r}\right)$ with $Q$ real into the equation $q_{t}=\mathcal{L} q$, we get with (3.7.b), (3.22), and (3.26)

$$
\Omega=\frac{Q^{2}}{k^{r}}\left[2\left(1+3 k^{2}\right)-4 i k Q-Q^{2}\right],
$$

so that

$$
\operatorname{Re} \Omega=\frac{Q^{2}}{k^{r}}\left[2+6\left(k^{r}\right)^{2}-6\left(k^{i}\right)^{2}+4 k^{i} Q-Q^{2}\right] .
$$

The term between square brackets has a maximum at $Q=2 k^{i}$, and at this value, we get

$$
\operatorname{Re} \Omega_{\max }=\frac{8\left(k^{i}\right)^{2}}{k^{r}}\left[1+3\left(k^{r}\right)^{2}-\left(k^{i}\right)^{2}\right] .
$$

Since points along the stable branch satisfy

$$
\operatorname{Im}(d \omega / d k)=k^{i}\left[1+3\left(k^{r}\right)^{2}-\left(k^{i}\right)^{2}\right]=0,
$$

we see that $\operatorname{Re} \Omega_{\max }=0$. Hence all modes of $\mathcal{L} q$ are stable except the one at $Q=2 k^{i}$, which is marginal. (Note that this perturbation corresponds to the first higher harmonic of the basic wave vector $k^{i}$ in the leading edge.) Together with the stabilizing effect of the first term on the right-hand side of Eq. (3.21) already discussed above this proves the stability of steady-state solution along the stable branch of the SH equation, and hence of the stability of its marginal stability point. Thus we have shown that the marginal stability point of the SH equation has a finite basin of attraction, which provides the after the fact justification for the numerical observation of Dee et al. ${ }^{5,6}$ that front solutions of this equation, growing out of localized initial conditions, propagate at the marginal stability velocity $v^{*}$. As also noted by Dee et al., 5,6 if the number of nodes passing through the front region is conserved-and this was found ${ }^{5,6}$ numerically - this allows one to determine the wavelength $\lambda$ of the emerging pattern from the relation $\lambda=2 \pi v^{*} / \operatorname{Im}\left[\omega\left(k^{*}\right)-v^{*} k^{*}\right]$.

Example III: the EFK equation. The algebra of this equation is quite analogous to that for the SH equation, and we obtain instead of (3.29) on the branch $v_{1}$ of Fig. 5 
$\operatorname{Re} \Omega=\frac{\gamma Q^{2}}{k^{r}}\left[-\gamma^{-1}+6\left(k^{r}\right)^{2}-6\left(k^{i}\right)^{2} 4 k^{i} Q-Q^{2}\right]$.

As discussed in Appendix B, $k^{i}=0$ on the branch $v_{1}$ (just as for the AE), and the marginal stability point is given by $\left(k^{*}\right)^{2}=(1-\sqrt{1-12 \gamma}) / 6 \gamma$ for $\gamma<\frac{1}{12}\left[\left(k^{i}\right)^{*}=0\right]$. Using this in (3.31), we see that the term in square brackets is always negative for all $k<k^{*}$; hence the eigenmodes of $\mathcal{L}$ are stable and the marginal stability point that exists on $v_{1}$ for $\gamma<1 / 12$ is attractive. Thus we predict that for $\gamma<1 / 12$ sufficiently localized initial conditions evolve into uniformly translating fronts whose speed approaches (see Appendix B)

$$
v_{1}^{*}=\frac{2}{\sqrt{54 \gamma}}\left[1+36 \gamma-(1-12 \gamma)^{3 / 2}\right]^{1 / 2},
$$

and whose appearance qualitatively resembles the front solutions found in the FK equation. Likewise, fronts which are initially such that $\phi(x, t=0)$ falls off exponentially but less fast than $\exp \left[-k^{*} x\right]$ will develop into uniformly translating fronts with speed $v>v^{*}$.

For $\gamma>\frac{1}{12}$, the situation is different. As we saw in Sec. III B and Fig. 5(d), the marginal stability point moves for $\gamma>\frac{1}{12}$ onto a different branch, labeled $v_{2}$, on which $\left(k^{i}\right)^{*}$ is nonzero. The other branch of solutions where $k^{i}=0$, labeled $v_{1}$, still exists and corresponds to smaller values of $k^{r}$, as can be seen clearly in the inset of Fig. 5(d). Moreover, the $v_{1}$ branch loses stability according to Eq. (3.31) at the point where $\left(k^{r}\right)^{2}=(6 \gamma)^{-1}$, and, as shown in Appendix $B$, this is precisely the point where the two branches $v_{1}$ and $v_{2}$ touch. The physical origin of the instability that develops along the $v_{1}$ branch can most easily be understood from Eq. (2.2). This equation shows that a solution is unstable if that solution does not correspond to a maximum in $k^{i}$ of the growth rate $\omega^{r}$ (for fixed $k^{r}$ ), in agreement with our picture of the front dynamics. Along the branch $v_{1}$ we find from $\omega=1+k^{2}-\gamma k^{4}$ that $\operatorname{Red}{ }^{2} \omega / d k^{2}=2\left[1-6 \gamma\left(k^{r}\right)^{2}\right]$. Thus, in agreement with Eq. (3.31), the branch where $k^{i}=0$ loses stability when $\left(k^{2}\right)>(6 \gamma)^{-1}$ because the growth rate of the leading edge solutions with $k^{i}=0$ and $\left(k^{r}\right)^{2}>\left(6 \gamma^{-1}\right)$ actually has a local minimum rather than a local maximum in the growth rate. The point where $\operatorname{Re} d^{2} \omega / d k^{2}=0$ is precisely the bifurcation point where $v_{2}$ splits off from $v_{1}$.

What do these results imply from fronts growing out of physically localized initial conditions for $\gamma>\frac{1}{12}$ ? Our physical picture is that the profile in the leading edge will first rapidly adjust so as to make the growth rate locally maximal for a given $q^{r}$; in other words, the initial dynamics is dominated by a rapid approach of $q\left(u^{r}, t\right)$ to a $k$ value on the stable branch while the subsequent $q$ dynamics is that of a slow evolution "along" this branch. Preliminary numerical work by Dee indicates that in line with this picture the region of the profile where initially $q^{r}\left(u^{r}, t=0\right)>(6 \gamma)^{-1 / 2}$ rapidly develops a nonzero imaginary component $q^{i}$, such that indeed $q$ becomes locally close to a $k$ value corresponding to the branch $v_{2}$ in Fig. 5(d). Along this stable branch, the nonlinear convective term discussed earlier operates as usual, and we therefore expect that the front velocity is driven towards the marginal stability value on $v_{2}$, given by (see Appendix B)

$v_{2}^{*}=\left(\frac{4}{3}+\frac{5}{18 \gamma}-\frac{1}{18 \gamma} \sqrt{7+24 \gamma}\right)\left(\frac{1+\sqrt{7+24 \gamma}}{24 \gamma}\right)^{-1 / 2}$.

Thus, if this general picture is correct, the asymptotic front velocity should again be given by a marginal stability expression for $\gamma>\frac{1}{12}$. However, the corresponding profile $\phi(x, t)$ cannot be a uniformly translating front solution anymore. The reason is the following: The counting argument of Appendix A shows that the asymptotic behavior of uniformly translating fronts generally falls off as $\exp (-k x)$ with $k$ the root of the equation $k v=\omega(k)$ corresponding to the smallest value of $k^{r}$. Thus, if a profile propagating at velocity $v_{2}^{*}$ were uniformly translating, its asymptotic behavior would not be given by $k_{2}^{*}$ but by the value $k_{1}$ corresponding to the point $\left(k_{1}, v_{1}=v_{2}^{*}\right)$ on the branch $v_{1}$, and hence it would be unstable. This is clearly inconsistent, and we have to conclude that the marginal stability front for $\gamma>\frac{1}{12}$ has to be an envelope front. The exact nature of the time dependence behind the leading edge in this case is beyond the scope of this analysis, and further numerical work is clearly needed.

Note also the interesting feature that according to these results, stable uniformly translating fronts do exist with $\left(k^{r}\right)^{2}<(6 \gamma)^{-1}$. Thus, if the initial profile $\phi(x, t=0)$ falls off as $\exp (-c x)$ with $c<(6 \gamma)^{-1 / 2}$, we expect such fronts to approach a uniformly translating front with a larger asymptotic speed $v_{1}\left(k^{r}=c\right)$. Only when $c>(6 \gamma)^{-1 / 2}$ do we expect the profiles to develop into envelope fronts.

This concludes the derivation of the marginal stability results within the context of our method. In the Sec. IV we will briefly touch on the major weaknesses of our approach and on some remaining open questions.

\section{CAVEATS, COMMENTS, AND OPEN QUESTIONS}

The approach we have developed is based on a number of assumptions. We now wish to call attention to some of these, as well as to some of the most important open problems. We also summarize the experimental tests of the theory.

(1) Experimental tests. Propagation of simple fronts whose dynamics is described by equations such as the FK equation (1.1) occurs in liquid crystals ${ }^{10-12}$ as well as chemical waves. ${ }^{57}$ Unfortunately, these experiments are at present not precise enough to put a stringent test to the theory. Experiments on fluid instabilities are apparently most suited to do so. A few years ago, Ahlers and Cannell ${ }^{9}$ performed an experiment on the propagation of a Taylor vortex front into a Couette flow after suddenly quenching the system above the threshold for the Taylor-Couette instability. Close to the threshold, such a system can be modeled by the amplitude equation, and hence these experiments provide a direct test of marginal stability for this equation. Quite surprisingly, 
however, Ahlers and Cannell ${ }^{9}$ found that the velocity of such fronts was about a factor of 2 smaller than predicted by the theory. In spite of detailed investigations, ${ }^{58}$ this discrepancy has not been resolved as yet. Very recent experiments in the same spirit by Fineberg and Steinberg, ${ }^{59}$ on the other hand, are more encouraging: Their results on the velocity of fronts in RayleighBénard cells are in excellent quantitative agreement with the theoretical predictions. Moreover, Fineberg and Steinberg also measure the wavelength of the rolls emerging behind the front. Since the SH equation differs only in the nonlinear terms from the proper amplitude equation, ${ }^{60}$ and since this difference does not affect the marginal stability prediction, this measurement offers an additional test of the theory. The fact that the observed wavelength is quite close to that predicted from the $\mathrm{SH}$ equation with the aid of marginal stability is therefore quite gratifying.

(2) The leading edge approximation. Our analysis is based exclusively on the behavior of the profile in the leading edge, and the question arises to what extent this part is representative for the whole profile. For the first-order partial differential equation studied by Shraiman and Bensimon, ${ }^{33}$ the leading edge is indeed representative: The solution of such equations can be obtained by the method of characteristics, and it is merely convenient, not necessary, to analyze the solution in the leading edge. For higher-order partial differential equations I expect the leading edge to be representative if there is a continuous family of stable front solutions. The idea is that a more complete analysis could in principle be done as follows. For a given stable front solution, the spectrum of eigenmodes of the linearized perturbations around this state contains slow modes associated with the presence of nearby stable front solutions; these will dominate the evolution of profiles that are close to a stable front solution. If one expands in these eigenfunctions, one expects the dynamics in the leading edge to be representative for the evolution of the eigenfunctions as a whole. In this picture, our analysis should be viewed as a short cut to obtain this behavior directly. A better assessment of the validity of this picture is needed, however, especially for fronts leading to periodic states.

As pointed out by Ben-Jacob et al., ${ }^{6}$ it may sometimes happen that a stable steady-state front solution decays faster in $x$ than would be expected on the basis of the leading edge analysis: This happens if the prefactor of the term $\exp (-k x)$ corresponding to smallest value of $k^{r}$ happens to vanish for that particular steady-state profile. However, the counting argument of Appendix A for uniformly translating solutions implies that this situation, which Ben-Jacob et al. ${ }^{6}$ refer to as "case II marginal stability," can only occur at discrete isolated values of the parameters. We will investigate the differences with case II marginal stability in a future publication.

(3) Existence of a continuum of front solutions. Clearly, the existence of a family of stable traveling front solutions is a necessary condition for our approach to apply; for, if there is not a continuous range of solutions, the particular solutions that do exist certainly depend on all (nonlinear) details of the profile. The well-known counting argument demonstrating the existence of a continuum of stable moving front solutions of Eq. (1.1) can easily be extended to uniformly translating solutions of the EFK equation and generalizations thereof (see Appendix A). However, I have not found a way to generalize such arguments to equations such as the $\mathrm{SH}$ equation whose relevant front solutions are not uniformly translating. Note that in these cases we actually expect the existence of a continuous two-parameter family of front solutions. For, if the stable state of the equation is periodic, it typically admits a continuous family of such stable solutions, parametrized, e.g., by their wavelength $\lambda$. For a fixed velocity $v$ and wavelength $\lambda$, one then expects by analogy that there exists a front solution. If this is true, variation of $v$ and $\lambda$ yields a two-parameter family of solutions. Recently, Collet and Eckmann ${ }^{40}$ have actually proven that this is indeed the case in the $\mathrm{SH}$ equation for small $\epsilon$. (The proof is highly nontrivial-in fact, even the very definition of such fronts is not straightforward.) Almost all of these will not satisfy $\operatorname{Im} d \omega / d k=0$ in the leading edge, and hence will be unstable. By imposing the condition Im $d \omega / d k=0$, we expect to find the continuous branches of stable solutions, parametrized by $v$, that are important for the marginal stability arguments. We also point out that our arguments and the fact that the $\mathrm{SH}$ equation reduces to the $\mathrm{AE}$ to lowest order of $\epsilon$ (see, e.g., Ref. 5) both indicate that there also is a two-parameter family of front solutions for this equation, but to our knowledge this has not yet been investigated.

(4) Effect of nonlinearities. According to the marginal stability theory, nonlinearities affecting the profile behind the leading edge are not important. To illustrate a possible effect of a nonlinearity that would still be consistent with the marginal stability theory, we consider Eq. (1.3) with $F(\phi)=d V / d \phi, F^{\prime}(0)=1$, and $V(\phi)$ of the form sketched in Fig. 7. The maximum of $V(\phi)$ labeled $S$ corresponds to a stable state and the local maximum $\boldsymbol{M}$ to a metastable state. As usual, we consider initially localized fronts propagating into the unstable state $U$. Since $F^{\prime}=d^{2} V / d \phi^{2}=1$, marginal stability predicts that the velocity of these fronts should approach $v^{*}=2$. However, from the analogy with a particle in a potential [cf. Eq. (1.2)], it is clear that we can always prevent the existence of a steady-state solution with $v=v^{*}=2$ connecting the states $U$ and $S$; indeed, by properly choosing the height of $V$ at $M$, we can ensure "trapping" in the local minimum between $M$ and $S$ for all "friction coefficients" $v>v_{c}$ (see, e.g., Fife ${ }^{13}$ for further discussion of such examples). This also implies that a front solution exists which connects $S$ and $M$ and which propagates with speed $v_{c}$. What will happen to fronts propagating into $U$ in this case? Since fronts leading to the emergence of the state $M$ always exists for all $v$, we in this case typically expect to find two fronts in the system: one connecting $U$ and $M$ and propagating at speed $v^{*}=2$ and a second slower one at speed $v_{c}<2$ connecting $M$ and $S$. A split up of this type has indeed been observed by Ben-Jacob et al. ${ }^{6}$ in numerical studies of a slightly different example. 


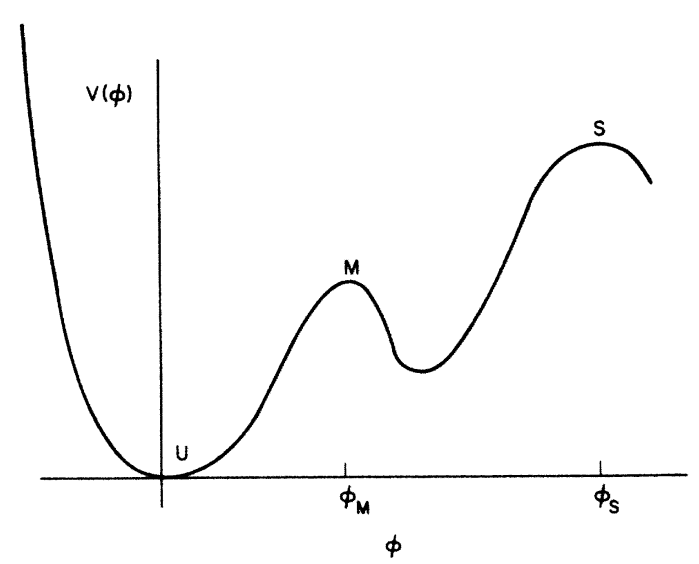

FIG. 7. The potential discussed in the text which is used to illustrate that a profile propagating into the unstable state $U$ $(\phi=0)$ can either give rise to the metastable state $\phi_{M}$ or the stable state $\phi_{S}$. At $\phi=0, d^{2} V / d \phi^{2}=1$.

(5) Selected wavelength does not minimize Lyapunov functional. All equations that we have discussed can be written in the form $\partial \phi / \partial t=-\delta F / \delta \phi$, where $F$ is a "free-energy" functional. As a result, $F$ also plays the role of a Lyapunov function, since $F$ cannot increase in time $\left[d F / d t=-(\delta F / \delta \phi)^{2} \leq 0\right]$. As pointed out already by Dee et al., ${ }^{5,6}$ however, the existence of a Lyapunov function does not appear to be relevant for front propagation into unstable states - indeed the wavelength of the periodic state emerging behind the front in the SH equation is different from the one that minimizes $F$. Another physically very different example where the dynamics drives a systems towards minimally stable states rather than a minimal energy state was recently discussed by Tang et al. ${ }^{61}$

(6) Bifurcation of unstable front solutions at the marginal stability point. For uniformly translating fronts of the form $\phi(x-v t)$, we were able to show that an unstable branch typically bifurcates off at the marginal stability point (see Sec. III B and Fig. 5). As a result, the stability of solutions as a function of $v$ is generally expected to be as sketched in Fig. 3(d). A simple way to analyze unstable fronts leading to periodic states is lacking, but apparently the situation is quite different here. Our arguments as well as the results of Collet and Eckmann ${ }^{40}$ described above suggest that there generally is a twoparameter family of solutions. Thus unstable states abound for all $v$, but since the stable solutions must satisfy the additional constraint (3.13), they will typically form a discrete set of one-parameter families of solutions (there can in principle be several branches). This has the following implications.

(7) Smallest $v^{*}$ versus smallest Rek*. Shraiman and Bensimon ${ }^{33}$ studied a first-order partial differential equation with a $v(k)$ curve (for first-order equations, $k$ is real) as sketched in Fig. 4(a), so that there are two marginal stability points, $v_{1}^{*}, k_{1}^{*}$ and $v_{2}^{*}, k_{2}^{*}$, with $v_{1}^{*}>v_{2}^{*}, k_{1}^{*}<k_{2}^{*}$. For sufficiently localized initial conditions, an argument similar to the one illustrated in Fig. 3(b) shows that the front velocity should approach the smallest $v^{*}$, i.e., $v_{2}^{*}$. For uniformly translating fronts, the situation is somewhat more subtle, however. In these cases, as we have discussed above, an unstable branch generally bifurcates off at the fixed point $v_{1}^{*}, k_{1}^{*}$. Since there is a one-parameter family of solutions, the existence of this branch will usually imply that the marginal stability point $v_{2}^{*}, k_{2}^{*}$ cannot represent the asymptotic decay of a fully nonlinear uniformly translating solution (it is "screened" by the unstable branch). If such fronts would remain uniformly translating, they will therefore have to propagate at velocity $v_{1}^{*}$. However, I consider it more likely that the front will usually change into an envelope front, since this allows its velocity to decrease to $v_{2}^{*}$ (cf. the discussion of the EFK equation in Sec. III C). For envelope fronts I similarly expect the smallest $v^{*}$ to be relevant in most cases, as found by Shraiman and Bensimon, ${ }^{33}$ since for envelope fronts the existence of a two-parameter family of front solutions opens the possibility that more than one branch can represent the leading edge behavior. Whether this indeed can happen deserves further study, but it appears that an educated guess can be made in a rather simple way for a given equation. Suppose the equation under study is known to allow periodic stable steady states for wavelengths $\lambda$ in the range $\lambda_{1} \leq \lambda \leq \lambda_{2}$. Assuming the validity of the empirical $1^{5,6}$ "conservation of nodes" rule we get, as discussed in Sec. III C, a relation between $\lambda$ and the parameters in the leading edge, $\lambda=2 \pi v /\left(\omega^{i}-v k^{i}\right)$. Together with the expression $v=\omega^{r} / k^{r}$, this allows us to obtain $k^{r}$ and $k^{i}$ as a function of $\lambda$. By varying $\lambda$ over the allowed range, we can then explicitly check which values of $k^{r}$ and $k^{i}$ are "accessible," i.e., represent the asymptotic behavior of global front solutions. Of the accessible marginal stability points, the one with the smallest velocity is then expected to give the asymptotic speed of most fronts growing out of sufficiently localized initial conditions. The existence of a finite basin of attraction of both points cannot, however, be excluded.

(8) Relevance of uniformly moving solutions with $k^{i} \neq 0$. The bifurcation analysis of Sec. III B showed that for uniformly translating fronts the marginal stability point on the branch where $k^{i}=0$ represents the smallest velocity at which $k^{i}=0$ in the leading edge. Also, when the marginal stability point moved onto a branch where $k^{i} \neq 0$, we saw that fronts would cease to be uniformly translating; this generally happens if (2.2) becomes violated. There is probably a deeper reason for the fact that only solutions with $k^{i}=0$ seem to give rise to uniformly translating fronts, but this question deserves further study. It is also of interest to study the AE with the same stabilizing fourth-order derivative as the EFK. The resulting equation allows periodic solutions, and such a study might therefore shed some light on points (6) and (7).

(9) Transients. As mentioned by Dee, ${ }^{8}$ in numerical studies the transients for the approach to $v^{*}$ are relatively short [the transient time is indeed ${ }^{23}$ of order unity for the FK equation (1.1) but transients decay algebraically rather than exponentially in time]. Our physical picture is based on the existence of two regimes for the transient behavior. For arbitrary initial conditions with a given 
$k^{r}$, the $k^{i}$ mode that grows fastest will rapidly drive $q$ towards a $k$ value corresponding to the stable branch. This is followed by a slower evolution of $q$ (algebraically in time) along the stable branch towards the marginal stability point. It would be of interest to study the latter transient behavior within the context of our approach.

(10) Extensions. In this paper we have confined the analysis to partial differential equations for a single field that are of first order in time but of arbitrary order in the spatial derivatives. Since marginal stability appears to work for certain more complicated cases as well, ${ }^{29}$ extensions of our approach are worth investigating: The case in which there is more than one field ${ }^{14}$ or in which there are higher-order time derivatives or more than one space dimension, as well as propagation into a periodic unstable state. ${ }^{8,62}$ Intuitively, however, it is not at all surprising that marginal stability has more general validity. As was discussed before, the front velocity $v\left(k^{r}\right)=\omega^{r} / k^{r}$ is generally expected to diverge in the limit $k^{r} \rightarrow 0$ for propagation into an unstable state (not for propagation into a metastable state). As a result, there will generally be a stable branch for $k^{r}$ small enough and $v$ large enough, and this branch will often end at a marginal stability point. If it does, the dynamical equation for $q$ will contain a nonlinear convection term that drives $q$ towards $k^{*}$ for sufficiently localized initial conditions.

(11) Further numerical work. Finally, we stress that more numerical studies would be extremely helpful to clarify the following issues.

The competition between initial conditions and noise (Sec. II).

The question of what happens if more than one marginal stability point is present [see point (6) above].

The propagation of fronts when the character of fronts is predicted to change, such as for the EFK equation at $\gamma=\frac{1}{12}$ (see sec. III C).

\section{CONCLUSIONS}

In this paper we have extended the marginal stability ideas, in the form sketched by Dee et al. ${ }^{5,6}$ and Shraiman and Bensimon, ${ }^{33}$ as far as possible. We have illustrated the physics underlying the marginal stability mechanism with analogies and have discussed how an equation that may be viewed as a generalization of the Burgers equation drives the front velocity to the marginal stability value, provided the front solutions lose stability because the group velocity in the tails becomes larger than the envelope velocity. The emerging picture is quite simple: for propagation into an unstable state, there generally is a branch of stable front solutions whose velocity $v\left(k^{r}\right)=\omega^{r} / k^{r}$ diverges for $k^{r} \rightarrow 0$. Thus, the velocity is increasing with the interface width, and since the effect of faster moving portions of the profile is to decrease its width, this also drives the velocity down towards the marginal stability value. For a given equation, we show how to check that the marginal stability fixed point is attractive by verifying the stability of $\mathcal{L}$. We have done so explicitly for the amplitude equation, the Swift-Hohenberg equation, ${ }^{63}$ and the extended Fisher-Kolmogorov equation. For the latter equation, the marginal stability point changes character at $\gamma=\frac{1}{12}$, and beyond this value localized initial conditions cannot develop into uniformly translating fronts. In spite of a number of open problems, our formulation together with the numerous (numerical) investigation by others give strong evidence for the ubiquity of this mechanism for propagation into an unstable state, and we hope that this work will help to establish that these ideas can be applied easily and with fairly high confidence to specific new problems.

\section{ACKNOWLEDGMENTS}

It is a pleasure to thank Pierre C. Hohenberg for stimulating discussions and Greg Dee for useful comments and for starting numerical work that guided my interpretation of the results for the EFK equation.

\section{APPENDIX A: EXISTENCE OF A CONTINUOUS FAMILY OF FRONT SOLUTIONS}

In this appendix we present a "counting argument" which shows the existence of a continuous oneparameter family of uniformly translating solutions $\phi(x-v t)$ for the particular class of equations for which $\omega(k)$ is a real polynomial in $k^{2}$. Uniformly traveling solutions will of course only exist for propagation of a stable state $\phi=\phi_{2}=$ const into an unstable state $\phi=\phi_{1}=$ const, as studied for the FK equation (1.1) and the EFK (3.2), and we therefore assume that the general equation admits such solutions.

Let $\omega_{1}(k)$ and $\omega_{2}(k)$ be the dispersion relation for perturbation of the form $e^{\omega t-k x}$ around the states $\phi_{1}$ and $\phi_{2}$, respectively. Since the state $\phi_{2}$ is assumed to be stable, $\omega_{2}\left(k=i k^{i}\right)<0$ for all $k^{i}$.

A profile uniformly translating the velocity $v$ corresponds to a solution of the nonlinear equation $-v(d \phi / d x)=F\left(\phi, \phi_{x x}, \ldots\right)$ with $\phi \rightarrow \phi_{2}$ for $x \rightarrow-\infty$ and $\phi \rightarrow \phi_{1}$ for $x \rightarrow \infty$. If the highest-order spatial derivative in $F$ is of order $N$ ( $N$ even), this equation corresponds to a set of first-order equations in a $N$ dimensional phase space $\left(\phi, \phi_{x}, \phi_{x x}, \ldots\right)$, and a uniformly translating front solution exists if there is a trajectory starting from the fixed point $\left(\phi_{2}, 0,0, \ldots\right)$ at $x=-\infty$ and flowing into the fixed point $\left(\phi_{1} 0,0, \ldots\right)$ for $x \rightarrow \infty$. In the neighborhood of the stable fixed point, the behavior of the eigenmodes $e^{-k x}$ is given by [cf. Eq. (3.17)]

$$
v k=\omega_{2}(k) \text {. }
$$

Consider first the solution of this equation for $v=0$. Since $\omega_{2}(k)=\omega_{2}(-k)$, the equation $\omega_{2}(k)=0$ has $N / 2$ roots with $k^{r}>0$ and $N / 2$ roots with $k^{r}<0$; Roots with completely imaginary $k$ are excluded because of the presumed stability of $\phi_{2}\left[\omega_{2}\left(k=i k^{i}\right)<0\right]$. Moreover, since $\omega_{2}$ is a real polynomial in $k^{2}$, we see that roots of Eq. (A1) can never cross the imaginary $k$ axis for $v=0$ [see Figs. 8(a) and 8(b)]. Hence, the fixed point $\left(\phi_{2}, 0,0, \ldots\right)$ has $N / 2$ stable and $N / 2$ unstable eigendirections for all $v$.

We now turn to the equation 


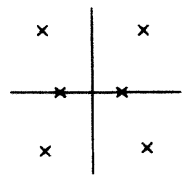

(a) $v=0$

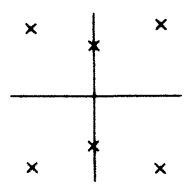

(d) $v=0$

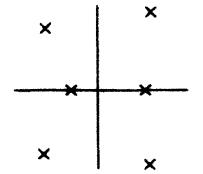

(b) $v>0$

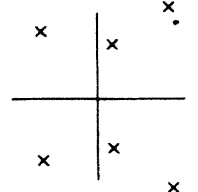

(e) $v>0$

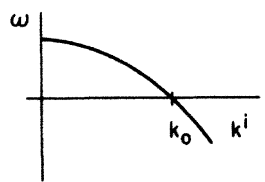

(c)

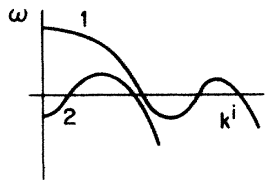

(f)
FIG. 8. (a) Qualitative sketch of the roots of the equation $\omega_{2}(k)=0$. (b) For $v \neq 0$, the location of the roots of Eq. (A1) is essentially as sketched in (a). (c) Qualitative behavior for purely imaginary values of $k\left(k=i k^{i}\right)$ of the function $\omega_{1}(k)$ discussed in the text. (d) Roots of the equation $\omega_{1}(k)=0$ for the function $\omega_{1}$ sketched in (c). (e) The roots of Eq. (A2) that lie on the imaginary axis for $v=0$ move into the right half-plane for $v \neq 0$. (f) Various possible behaviors of the function $\omega_{1}\left(i k^{i}\right)$ discussed in the text.

$$
v k=\omega_{1}(k),
$$

which determines the eigenvalues around the fixed point $\left(\phi_{1}, 0,0, \ldots\right)$. Since the state $\phi_{1}$ is unstable, $\omega_{1}(k)$ must be positive for some range of $k$ values along the imaginary axis, and since for physical reasons $\omega_{1}(k)$ is generally negative for large imaginary values of $k, \omega_{1}$ must be zero at some points on the imaginary $k$ axis. We first discuss the typical case in which $\omega_{1}\left(k=i k^{i}\right)$ is of the form sketched in Fig. 8(c), so that for $v=0$, Eq. (A2) has two roots at $k= \pm i k_{0}$, say, on the imaginary $k$ axis [Fig. $8(\mathrm{~d})$ ]. The location of these roots for small $v$ can be studied by expanding Eq. (A2) around $v=0, k= \pm i k_{0}$. One then finds that the imaginary part $\left(k^{i}-k_{0}\right)$ is of order $v^{2}$, while $k^{r}$ of the two roots is according to the imaginary part of Eq. (A2) to order $v$ given by

$$
k^{r}=\frac{v k_{0}}{\left.\operatorname{Im} \frac{d \omega}{d k}\right|_{k=i k_{0}}}, \quad k^{r}=\frac{-v k_{0}}{\left.\operatorname{Im} \frac{d \omega}{d k}\right|_{k=-i k_{0}}} .
$$

Now, Im $d \omega / d k=-\partial \omega^{r} / \partial k^{i}$ (Cauchy-Riemann), and according to the behavior sketched in Fig. 8(c), $\partial \omega^{r} / \partial k^{i}<(>) 0$ at $k=+(-) i k_{0}$. Taken together, these results show that for both roots, $k^{r}>0$ for $v>0$, i.e., they both move into the right half-plane. Since roots cannot cross the imaginary axis for $v \neq 0$, the location of roots is as sketched in Fig. 8(e) for all $v \neq 0$, implying that the fixed point $\left(\phi_{1}, 0,0, \ldots\right)$ has $\frac{1}{2} N+1$ stable eigen directions $\left(k^{r}>0\right)$ and $\frac{1}{2} N-1$ unstable ones.

A simple counting argument now shows the existence of a uniformly translating solution for any $v$. An arbitrary trajectory leaving the fixed point $\left(\phi_{2}, 0,0, \ldots\right)$ will not flow into the other fixed point, since it will in general be on the $\frac{1}{2} N-1$-dimensional unstable manifold of $\left(\phi_{1}, 0,0, \ldots\right)$. The requirement that a trajectory does not lie on this manifold (and hence approaches this fixed point) is expressed by $\frac{1}{2} N-1$ condition. The existence of $\frac{1}{2} N$ unstable direction at $\left(\phi_{2}, 0,0, \ldots\right)$, on the other hand, gives precisely the necessary $\frac{1}{2} N-1$ free parameters to be able to obey these conditions, and hence to find a particular trajectory that connects the two fixed points. This shows the existence of a solution for any $v$.

The above analysis applies directly to the EKF equation, where $\omega$ is indeed of the form sketched in Fig. 8(c). Clearly, the analysis also shows the existence of a family of solutions for $\omega\left(k=i k^{i}\right)$ of the form of curve 1 in Fig. $8(\mathrm{f})$, as long as $\omega(0)>0$ and $\omega(k \rightarrow \pm i \infty)<0$, since this is sufficient to ensure that $\left(\phi_{1}, 0,0, \ldots\right)$ has two more stable directions than unstable ones. When $\omega(0)<0$, on the other hand, there is not a continuous range of solutions $\phi(x-v t)$. This is what one would expect physically, however, since in this case $\phi_{1}$ is only unstable against certain finite wavelength perturbations [see Fig. 8(f), curve 2], and in this case one expects an envelope front rather than a uniformly translating one to propagate into $\phi_{1}$. Moreover, the stability arguments presented at the end of Sec. III C indicated that uniformly translating solutions are only relevant for $\omega(k)$ of the form sketched in Fig. 8(c), whose growth rate is maximal at $k^{i}=0$.

As in Sec. III B where the unstable branch could not be investigated for envelope fronts, we cannot extend the above counting argument in a straightforward way to fronts leading to periodic solutions, as these are not described by a simple time-independent differential equation. Nevertheless, intuitively one expects many of the features we found above to be rather general. For instance, as the discussion in Sec. III B illustrates, uniformly translating solutions are more restricted than solutions whose envelope is moving with constant speed, indicating that the latter class of solutions is larger than the first and at least contains a continuous family of solutions. As argued in Sec. IV, the additional freedom associated with the wavelength $\lambda$ of the stable states then will in general give rise to a two-parameter family of solutions. For propagation leading to periodic states whose wavelength is short compared to the front width, it might be possible to show this using a multiple-scale argument. $^{48,60}$

\section{APPENDIX B: RESULTS FOR THE EFK EQUATIONS}

For the EFK equation (3.2), $\omega(k)$ is, according to (3.4c), given by

$$
\omega=1+k^{2}-\gamma k^{4} \text {. }
$$

First consider the stable branch for which, according to Eq. (3.13), $\operatorname{Im} d \omega / d k=0$. This equation is trivially satisfied by taking $k^{i}=0$. On this branch, Eq. (3.10) then gives for the velocity

$$
v\left(k^{r}\right)=\left[1+\left(k^{r}\right)^{2}-\gamma\left(k^{r}\right)^{4}\right] / k^{r} .
$$

For $\gamma<\frac{1}{12}$, this velocity has a minimum at

$$
\left(k^{r}\right)^{2}=[1-\sqrt{1-12 \gamma}] / 6 \gamma .
$$


This minimum corresponds to the marginal stability point, and the corresponding marginal stability velocity is given by

$$
v_{1}^{*}=\frac{2}{\sqrt{54 \gamma}}\left[1+36 \gamma-(1-12 \gamma)^{3 / 2}\right]^{1 / 2}, \quad \gamma<\frac{1}{12} .
$$

There is a second branch of solutions of Eq. (3.13) for which $k^{i} \neq 0$. For this branch, (3.13) yields with (B1)

$$
\left(k^{i}\right)^{2}=3\left(k^{r}\right)^{2}-(2 \gamma)^{-1} \text {. }
$$

Clearly, these solutions only exist for $\left(k^{r}\right)^{2}>(6 \gamma)^{-1}$. Substitution of this result into Eq. (3.10) yields for the corresponding velocity

$$
\begin{array}{r}
v_{2}\left(k^{r}\right)=\left[1+(4 \gamma)^{-1}-2\left(k^{r}\right)^{2}+8 \gamma\left(k^{r}\right)^{4}\right] / k^{r}, \\
\left(k^{r}\right)^{2}>(6 \gamma)^{-1} .
\end{array}
$$

Equations (B2) and (B6) yield

$$
v_{2}-v_{1}=\frac{9 \gamma}{k^{r}}\left[\frac{1}{6 \gamma}-\left(k^{r}\right)^{2}\right]^{2} .
$$

Hence the second branch bifurcates off of the first one at $\left(k^{r}\right)^{2}=(6 \gamma)^{-1}$ and corresponds to higher velocities. Comparison with the discussion following Eq. (3.32) shows that this is also the point where the branch $v_{1}$ loses stability. The branch $v_{2}$ given by (B6) can also have a minimum at

$$
\left(k_{2}^{r}\right)^{*}=\left[\frac{1}{6 \gamma} \frac{(1+\sqrt{7+24 \gamma})}{4}\right]^{1 / 2}, \gamma>\frac{1}{12}
$$

and this corresponds to the marginal stability point on the branch $v_{2}$. From Eqs. (B6) and (B8), one easily obtains Eq. (3.33). According to Eqs. (B4) and (B8), the EFK always has one marginal stability point given by Eq. (1.5), but for $\gamma>\frac{1}{12}$ this point lies on the branch $v_{2}$.

The uniformly translating solutions $\phi(x-v t)$ satisfy Eq. (3.17). The imaginary part of this equation yields

$$
v k^{i}=2 k^{i} k^{r}\left[1-2 \gamma\left(k^{r}\right)^{2}+12 \gamma\left(k^{i}\right)^{2}\right] \text {. }
$$

The solution $k^{i}=0$ corresponds to the stable branch already analyzed above. The unstable branch of solutions is therefore the one at which

$$
\left(k^{i}\right)^{2}=\left[v / k^{r}-2+4 \gamma\left(k^{r}\right)^{2}\right] / 4 \gamma .
$$

Substitution of this result into the real part of Eq. (3.17) then yields after some algebra

$$
v=2 k^{r}\left[1+4 \gamma-8 \gamma\left(k^{r}\right)^{2}+16 \gamma^{2}\left(k^{r}\right)^{4}\right]^{1 / 2},
$$

with the constraint that the term in square brackets in (B10) be positive. It may be checked that for $\gamma<\frac{1}{12}$ this unstable branch (indicated by a dotted line in Fig. 5) intersects the stable one at the marginal stability point, as predicted in Sec. III B. For $\gamma>\frac{1}{12}$, the two branches intersect at some $k^{r}>(6 \gamma)^{-1 / 2}$, so that according to the discussion in Sec. III C the branch $v_{1}$ loses stability before it is intersected by the unstable branch (B11). This point at which the upper branch loses stability for $\gamma=\frac{1}{4}$ is indicated by a cross in Fig. 5(d). It is also the point where the branch $v_{2}$ bifurcates off of the $v_{1}$ branch. 1(a) A short version of this work was published in W. van Saarloos, Phys. Rev. Lett. 58, 2571 (1987); (b) R. A. Fisher, Ann. Eugenics 7, 355 (1937).

${ }^{2}$ A. Kolmogorov, I. Petrovsky, and N. Piskunov, Bull. Univ. Moskou, Ser. Internat., Sec. A 1, 1 (1937).

${ }^{3} \mathrm{An}$ overview of these models and references to this literature can, e.g., be found in the three separate papers by D. G. Aronson, E. D. Conway, and H. F. Weinberger, in Partial Differential Equations and Dynamical Systems, edited by W. E. Fitzgibbon III (Pitman, Boston, 1984).

${ }^{4}$ A. C. Scott, Neurophysics (Wiley, New York, 1977); Rev. Mod. Phys. 47, 487 (1975).

${ }^{5}$ G. Dee and J. S. Langer, Phys. Rev. Lett. 50, 383 (1983).

${ }^{6}$ E. Ben-Jacob, H. R. Brand, G. Dee, L. Kramer, and J. S. Langer, Physica (Utrecht) 14D, 348 (1985); some aspects of the analysis in this paper are along the lines of those of Scott (see his book in Ref. 4).

${ }^{7}$ For an introduction and review, see, e.g., P. C. Hohenberg and M. C. Cross, in Fluctuations and Stochastic Phenomena in Condensed Matter, edited by L. Garrido (Springer, New York, 1987). The same authors are writing a more detailed review.

${ }^{8}$ This way of creating front propagation is discussed in some more detail by G. Dee, J. Stat. Phys. 39, 705 (1985); see also G. Dee, Physica (Utrecht) 15D, 295 (1985).

${ }^{9}$ G. Ahlers and D. S. Cannell, Phys. Rev. Lett. 50, 1583 (1983).

${ }^{10}$ L. Leger, Solid State Commun. 11, 1499 (1972), Mol. Cryst.
Liq. Cryst. 24, 33 (1973). X. Y. Wang, Phys. Lett. 112A, 402 (1985); Phys. Rev. A 32, 3126 (1985); 34, 5179 (1986); L. Lin and Ch.-Q. Shu, Phys. Lett. 119A, 178 (1986).

${ }^{11}$ G. Zhu, Phys. Rev. Lett. 49, 1332 (1982) X. Y. Wang, Phys. Lett. 98A, 259 (1983); Commun. Theor. Phys. 2, 1307 (1983); L. Lin, Ch.-Q. Shu, and X. Gang, J. State. Phys. 39, 633 (1985).

${ }^{12}$ P. E. Cladis, H. R. Brand, and P. L. Finn, Phys. Rev. A 28, 512 (1983), and (to be published); J. E. Maclennan, M. A. Handschy, and N. A. Clark, Phys. Rev. A 34, 3554 (1986).

${ }^{13}$ See, e.g., P. Fife, Mathematical Aspects of Reacting and Diffusing Systems, Vol. 28 of Lecture Notes in Biomathematics, edited by S. Levin (Springer, New York, 1979); J. Smoller, Shock Waves and Reaction-Diffusion Equations (Springer, New York, 1983).

${ }^{14}$ J. P. Keener, SIAM J. Appl. Math. 39, 528 (1980).

${ }^{15}$ See, e.g., Oscillations and Traveling Waves in Chemical Systems, edited by R. J. Field and M. Burger (Wiley, New York, 1985); P. Fife, in Nonequilibrium Cooperative Phenomena in Physics and Related Fields, edited by M. G. Velarde (Plenum, New York, 1984).

${ }^{16} \mathrm{~A}$ review with many references to the Russian literature is given by Ya. B. Zel'dovich and B. A. Malomed, Izv. Vyssh. Uchebn. Zaved. Radiofiz 25, 591 (1982) [Radiophys. Quantum Electron. 25, 421 (1982)]; cf. also B. A. Malomed and A. M. Zhabotinskii, in Nonlinear and Turbulent Processes, edited by R. Z. Sagdeev (Gordon and Breach, New York, 1984). 
${ }^{17}$ I. M. Gel'fand, Usp. Mat. Nauk. 14, No. 2 (86), 87 (1959) [Am. Math. Soc. Transl. Ser. 2, 29, 295 (1963)]; Ya. B. Zel'dovich and G. I. Barenblatt, Combust. Flame 3, 61 (1959).

${ }^{18}$ M. Nauenberg, Phys. Rev. B 28, 449 (1983).

${ }^{19}$ M. Nauenberg, R. Richter, and L. M. Sander, Phys. Rev. B 28, 1650 (1983).

${ }^{20}$ G.Parisi and Y. C. Zhang, J. Stat. Phys. 41, 1 (1985).

${ }^{21}$ D. Bensimon, B. Shraiman, and L. P. Kadanoff, in Kinetics of Aggregation and Gelation, edited by F. Family and D. P. Landau (Elsevier-North Holland, Amsterdam, 1984).

${ }^{22}$ D. G. Aronson and H. F. Weinberger, in Partial Differential Equations and Related Topics, edited by J. A. Goldstein (Springer, Heidelberg, 1975); Adv. Math. 30, 33 (1978); see also $\mathrm{H}$. Weinberger, Ref. 3, and references therein.

${ }^{23}$ For a discussion of the rate of approach of the speed to its asymptotic value see M. Bramson, Mem. Am. Math. Soc. 285, 1 (1983) and references therein.

${ }^{24} \mathrm{H}$. F. Weinberger, in Ref. 3. For a discussion of the convergence of discrete stochastic models to the continuum equations see M. Bramson, P. Calderoni, A. De Masi, P. Ferrari, J. L. Lebowitz, and R. H. Schonmann, J. Stat. Phys. 45, 905 (1986) and references therein.

${ }^{25}$ O. Diekman, J. Diff. Equations 33, 58 (1979).

${ }^{26}$ This concept of marginal stability was first discussed in the context of dendritic growth. See J. S. Langer and $\mathbf{H}$. Müller-Krumbhaar, Acta Metall. 26, 1681 (1978); 26, 1689 (1978); 26, 1697 (1978); J. S. Langer, Rev. Mod. Phys. 52, 1 (1980).

${ }^{27}$ Hence the "natural" asymptotic speed $v$ " is the slowest one possible. This seemingly counterintuitive result will be explained in Sec. II.

${ }^{28}$ The idea that the leading edge is the most relevant part of the profile for the type of front propagation discussed here already emerged in a paper by J. S. Langer and $H$. MüllerKrumbhaar, Phys. Rev. A 27, 499 (1983).

${ }^{29}$ Dee (Ref. 8) discusses an example where the marginal stability result (1.5) also applies to the propagation of fronts into a periodic unstable state.

30J. Swift and P. C. Hohenberg, Phys. Rev. A 15, 319 (1977); the equation is referred to in Ref. 6 as the Swift-HohenbergPomeau-Manneville equation since it was also studied in the context of pattern formation by Y. Pomeau and P. Manneville, Phys. Lett. 75A, 296 (1980). See also L. Kramer and P. C. Hohenberg, Physica (Utrecht) 13D, 357 (1984).

${ }^{31}$ Note in this respect that the proof of Aronson and Weinberger (Ref. 22) relies on the use of a maximum principle for positive solutions of Eq. (1.3) (see also P. Fife, Ref. 13). For the Swift-Hohenberg equation, the pattern emerging behind the front is not positive everywhere and the maximum principle cannot be applied.

${ }^{32}$ In view of the present zeitgeist, it seems appropriate to stress that we advocate this connection here only for the welldefined class of problems where there is a continuous range of steady-state front solutions, parametrized by their (envelope) velocity $v$. This generally is the case for propagation into an unstable state but not for propagation into a metastable state. See Secs. III and IV for further discussion of this point.

${ }^{33}$ B. Shraiman and D. Bensimon, Phys. Scr. T9, 123 (1985).

${ }^{34}$ See in particular the work by Keener (Ref. 14), who discussed coupled equations of the form $\epsilon \partial u / \partial t=D_{1} \nabla^{2} u$ $+f(u, v), \partial v / \partial t=\epsilon^{2} D_{2} \nabla^{2} v+g(u, v), \epsilon \ll 1$. Note also that the Swift-Hohenberg equation (1.6) can be written as $\partial u / \partial t=\nabla^{2} v+v+\epsilon u-u^{3}, \quad \nabla^{2} u+v=0$, indicating that it may be possible to analyze this equation following the methods discussed by Keener. I thank P. C. Hohenberg for pointing this out to me.

${ }^{35}$ H. J. Mikeska, J. Phys. C 11, L29 (1978); for a general review of magnetic domain walls see, e.g., F. H. de Leeuw, R. van den Doel, and U. Enz., Rep. Prog. Phys. 43, 689 (1980); V. G. Bar'yakhtar, B. A. Ivanov, and M. V. Chetkin, Usp. Fiz. Nauk. 146, 417 (1985); [Sov. Phys._Usp. 28, 563 (1985)].

${ }^{36}$ For a review, see, e. g., M. A. Collins, in Advances in Chemical Physics, edited by I. Prigogine and S. A. Rice (Wiley, New York, 1983), Vol. 53.

${ }^{37}$ This large friction regime is the regime $\gamma W / v \gg 1$, where $W$ is the wall width and $v$ the wall velocity.

${ }^{38}$ Although walls in liquid crystals are sometimes called solitons, it should be kept in mind that the term soliton usually refers to traveling waves which maintain their shape following interaction with other solitons. [See, e.g., G. L. Lamb, Jr., Elements of Soliton Theory (Wiley, New York, 1980).] In the high friction limit relevant for liquid crystals, such waves do not exist.

${ }^{39}$ See, e.g., P. G. de Gennes, The Physics of Liquid Crystals (Oxford University Press, Oxford, 1974).

${ }^{40}$ The existence of a continuous family of steady-state solutions appears to be a general feature of front propagation into an unstable state, but we have not been able to prove this in general. For the Swift-Hohenberg equation (1.6), a twoparameter continuous family of traveling-wave solutions was proven to exist in a certain parameter range by $P$. Collet and J. P. Eckmann, Commun. Math. Phys. 107, 39 (1986); Physica (Utrecht) 140A, 96 (1986). Presumably, this implies that there is a one-parameter family of stable front solutions. See Sec. IV and Appendix A for further discussion.

${ }^{41}$ This analogy with crystal growth was emphasized to me by G. H. Gilmer and J. D. Weeks (private communication).

${ }^{42}$ The growth form of crystals whose interface is rough was first discussed by F. C. Frank, in Growth and Perfection of Crystals, edited by R. H. Doremus, B. W. Roberts, and D. Turnbull (Wiley, New York, 1959).

${ }^{43}$ Sharp shocks can and do of course occur in the first-order partial differential equations studied by Shraiman and Bensimon (Ref. 33). In fact, Fig. 3(b) was inspired by their Fig. 2.

${ }^{44}$ Clearly, steady-state solutions can also change stability if their structure changes at a certain point, so that, e.g., condition (2.2) below becomes violated. This will prevent the marginal stability point defined by Eq. (1.5) from being approached. This happens for uniformly translating front solutions for $\gamma>\frac{1}{12}$ in the extended FK equation to be discussed in Sec. III. The analysis of this equation suggests that such a change of stability is often accompanied by a change in structure at the marginal stability point.

${ }^{45}$ See, e.g., G. B. Whitham, Linear and Nonlinear Waves (Wiley, New York, 1974).

${ }^{46}$ In fact, in the numerical studies of Ref. 33, the creation of fronts with a higher velocity via the judicious choice of initial conditions required careful programming since small errors tended to drive the velocity to the marginal stability value [D. Bensimon (private communication)].

${ }^{47}$ These results can be obtained as follows. By linearizing the equation in Fig. 2(a), one finds that $\phi(x, t=0)$ drops off as $\exp \left[-H_{i}\left(\chi_{a} / K_{2}\right)^{1 / 2} x\right]$; by transforming the equation of Fig. 2(b) to the dimensionless form of the FK equation, one sees that $v^{*}=2\left(\chi_{a} K_{2} / \gamma^{2}\right)^{1 / 2} H_{f}$ and the corresponding asymptotic behavior of $\phi$ is 


$$
\exp \left[-H_{f}\left(\chi_{a} / K_{2}\right)^{1 / 2}\left(x-v^{*} t\right)\right] .
$$

Since $\phi(x, t=0)$ drops off slower in $x$ than the marginal stability expression for $H_{i}<H_{f}$, the initial conditions are important in this regime. The front speed in this regime is then obtained by substituting

$$
\phi(x, t) \approx \exp \left[-H_{i}\left(\chi_{a} / K_{2}\right)^{1 / 2}(x-v t)\right]
$$

into the linearized form of the equation of Fig. 2(b).

${ }^{48}$ A. C. Newell and J. W. Whitehead, J. Fluid Mech. 38, 279 (1969); L. A. Segel, ibid. 38, 203 (1969).

${ }^{49} \mathrm{We}$ have omitted odd derivatives $\phi_{x}, \phi_{x x x}$, etc., from $F$ since in cases with spatial reflection symmetry these terms are usually absent from the linearized equation (they can occur in the form $\phi_{x}^{2} / \phi^{2}$, though - see, e.g., the model studied in Ref. 33). The analysis in Sec. III is unaffected by the presence of such terms in $F$, but it should be kept in mind that according to the analysis presented Appendix A, there may not always be a continuous family of steady-state front solutions in the absence of reflection symmetry.

${ }^{50}$ For an analytic function $\omega(k)$, the Cauchy-Riemann equations express the fact that the derivative $d \omega / d k$ is unique and independent of the way in which the variation is taken. This implies $\operatorname{Re} d \omega / d k=\partial \omega^{r} / \partial k^{r}$ and also, at the points where (3.13) holds, $\partial \omega^{r} / \partial k^{i}=-\partial \omega^{i} / \partial k^{r}=0$. In considering $v\left(k^{r}\right)$ in (3.15), it is understood that $k^{i}$ is expressed as a function of $k^{r}$ with the aid of (3.13), so that $\omega^{r}$ stands for $\omega^{r}\left[k^{r}, k^{i}\left(k^{r}\right)\right]$ and $d \omega^{r} / d k^{r}$ for $\partial \omega^{r} / \partial k^{r}+\partial \omega^{i} / \partial k^{r}\left(d k^{i} / d k^{r}\right)$. Using the above two results based on the analyticity of $\omega(k)$, we then get in (3.15) $d \omega^{r} / d k^{r}=\operatorname{Re} d \omega / d k$.

${ }^{51}$ Thus these considerations do not apply to LandauGinzburg-type equations with complex coefficients, which arise, e.g., in the theory of chemical waves [see, e.g., Y. Kuramoto, Chemical Oscillations, Waves and Turbulence (Springer, New York, 1984)].

${ }^{52}$ This generalizes the observation (see, e.g., Ref. 6) that the solutions of Eq. (1.3) become oscillatory in space for velocities below the marginal stability value.

${ }^{53} \mathrm{~A}$ similar independent variable transformation is also useful for the discussion of the stability of solitary waves. See E. Magyari, Phys. Rev. A 31, 1174 (1985).

${ }^{54}$ J. M. Burgers, The Nonlinear Burgers Equation (Reidel, Boston, 1974).

${ }^{55}$ In Fig. 6(a), it is assumed that $q^{r}$ intersects the line $q^{r}=\left(k^{r}\right)^{*}$. From the analysis in the text, it therefore would appear that solutions with $q^{r}>\left(k^{r}\right)^{*}$ initially for all $u^{r}$ would approach steady-state solutions with $k^{r}>\left(k^{r}\right)^{*}$. This is generally not the case, however, since such solutions are unstable and since, as argued in Sec. III B, such $k$ values generally do not represent the asymptotic decay of the profile.

${ }^{56}$ In connection with the possibility sketched in Fig. 6(b), we remark that there is a possibly important difference between first-order partial differential equation and higher-order partial differential equations. As noted by Bensimon and Shraiman (Ref. 33), if $q$ (which is real for first-order equations) is less than $k_{\max }$ initially, then $q<k_{\max }$ for all times. This already implies the result sketched in Fig. 6(b). For higherorder partial differential equations, such a result generally does not hold, and, although this does not happen for the case studied by Aronson and Weinberger, one apparently cannot exclude the possibility that in certain cases values of $q^{r}>k_{\max }$ are generated if initially $q^{r}<k_{\max }$. If this happens, I would expect this to drive $v$ towards $v^{*}$ again.

${ }^{57}$ This subject will be reviewed by Hohenberg and Cross (Ref. 7); see also the article by Saul and Showalker, in the book by Field and Burger, Ref. 15.

${ }^{58}$ M. Lücke, M. Mihelcic, and K. Wingerath, Phys. Rev. Lett. 52, 625 (1984); Phys. Rev. A 31, 396 (1985); M. Lücke, M. Mihelcic and B. Kowalski, Phys. Rev. A 35, 4001 (1987); M. Lücke, M. Mihelcic, B. Kowalski, and K. Wingerath, in The Physics of Structure Formation: Theory and Simulation, edited by W. Güttinger and G. Dangelmayr (Springer, New York, 1987).

${ }^{59}$ J. Fineberg and V. Steinberg, Phys. Rev. Lett. 58, 1332 (1987).

${ }^{60}$ M. C. Cross, Phys. Fluids 23, 1727 (1980).

${ }^{61}$ C. Tang, K. Wiesenfeld, P. Bak, S. Coppersmith, and P. Littlewood, Phys. Rev. Lett. 58, 1161 (1987).

${ }^{62}$ To some extent, these extensions are all more or less related. For instance, the problem of propagation into a periodic unstable state of the AE can, as shown by Dee (Ref. 8), be transformed into the problem of propagation into a homogeneous unstable state of a pair of equations for two fields.

${ }^{63} \mathrm{~A}$ detailed mathematical proof for the Swift-Hohenberg equation has recently been given by P. Collet and J.-P. Eckmann, Helv. Phys. Acta (to be published). 